\title{
Nonparametric Tests for Treatment Effect Heterogeneity with Duration Outcomes
}

\author{
Pedro H. C. Sant'Anna* \\ Vanderbilt Univeristy
}

\begin{abstract}
This article proposes different tests for treatment effect heterogeneity when the outcome of interest, typically a duration variable, may be right-censored. The proposed tests study whether a policy 1) has zero distributional (average) effect for all subpopulations defined by covariate values, and 2) has homogeneous average effect across different subpopulations. The proposed tests are based on two-step Kaplan-Meier integrals and do not rely on parametric distributional assumptions, shape restrictions, or on restricting the potential treatment effect heterogeneity across different subpopulations. Our framework is suitable not only to exogenous treatment allocation but can also account for treatment noncompliance - an important feature in many applications. The proposed tests are consistent against fixed alternatives, and can detect nonparametric alternatives converging to the null at the parametric $n^{-1 / 2}$-rate, $n$ being the sample size. Critical values are computed with the assistance of a multiplier bootstrap. The finite sample properties of the proposed tests are examined by means of a Monte Carlo study and an application about the effect of labor market programs on unemployment duration. Open-source software is available for implementing all proposed tests.
\end{abstract}

Keywords: Causal inference; duration data; empirical process; Kaplan-Meier; survival analysis

${ }^{*}$ Date: February 8 2020. Contact information: Department of Economics, Vanderbilt University, VU Station B \#351819, 2301 Vanderbilt Place, Nashville, TN 37235-1819 (e-mail: pedro.h.santanna@vanderbilt.edu). Financial support from the Spanish Plan Nacional de I+D+I (Grant No. ECO2012-33053 and ECO2014-55858$\mathrm{P})$ is acknowledged. This article is a revised version of the earlier draft entitled "Nonparametric Tests for Conditional Treatment Effects with Duration Outcomes", which is part of the author's dissertation "Essays on Duration and Count Data Models,' Universidad Carlos II de Madrid in Madrid, Spain, which won the 2016 Zellner Award, sponsored by the ASA Section on Business and Economic Statistics and SAS. The author thanks Miguel Delgado, Carlos Velasco, Winfried Stute, Juan Carlos Escanciano, Tong Li, Atsushi Inoue, and seminar participants at many seminars/conferences for helpful comments and discussions. 


\section{Introduction}

Assessing whether a policy or treatment has any effect on an outcome of interest has been one of the main concerns in economics and statistics. As summarized by Imbens and Wooldridge (2009), the focus of policy evaluation literature has been mainly confined to identifying and estimating unconditional treatment effect (TE) measures such as the average, distribution and quantile treatment effects. However, one important aspect of policy evaluations is that treatment effects tend to vary across different subpopulations, and focusing on unconditional TE measures can mask important heterogeneity in policy interventions. For instance, a labor market program that does not affect the unemployment duration for the overall population might still be effective for a subgroup of individuals with specific observable characteristics. Assessing if this is the case is particularly important for researchers and policymakers interested in generalizing some findings across time, places and populations, which the literature calls "external validity"; see e.g. Hotz et al. (2005), Bitler et al. (2006, 2008, 2016), Crump et al. (2008), and Ding et al. (2015). Treatment effect heterogeneity also plays an important role in designing statistical treatment rules, see e.g. Manski (2004).

In this article, we propose a unified approach to construct tests for different forms of treatment effect heterogeneity, paying particular attention to situations in which the outcome of interest, typically a duration variable, may be subject to right censoring. We develop tests for both average and distribution treatment effects conditional on covariate values. In particular, we consider nonparametric tests to assess whether $(a)$ there is any particular subpopulation defined by covariates for which a policy intervention has a nonzero distribution (or average) effect, and (b) the average treatment effect vary across different subgroups. All proposed tests can be applied under unconfounded treatment assignments, see e.g. Rosenbaum and Rubin (1983), but also when selection into treatment is endogenous and a binary instrumental variable is available to the researcher, see e.g. Imbens and Angrist (1994) and Angrist et al. (1996). Finally, we emphasize that all proposed tests are also directly applicable to assess TE heterogeneity with respect to a subset of the available covariate and not only with respect to the entire vector of observed characteristics; see Remark 1.

The proposed methodology relies on three main components. First, the tests are based on inverse probability weighted (IPW) estimators, in which the propensity score is estimated by nonparametric methods. We focus on the Series Logit Estimator proposed by Hirano et al. (2003). Second, as we are interested in TE heterogeneity across subgroups defined by covariates, 
the tests are based on conditional moment restrictions. To avoid the use of smooth estimates and the "curse of dimensionality," we adopt an integrated moment approach, see e.g. Bierens (1982), Bierens and Ploberger (1997), Stute (1997), and Escanciano (2006). Finally, to tackle the potential censoring problem inherited in duration outcomes, we characterize the integrated moments as Kaplan-Meier (KM) integrals, see e.g. Stute and Wang (1993), Stute (1993, 1995, 1996), Chen and Lo (1997), Sellero et al. (2005), and Sant'Anna (2016). It is important to emphasize that such an approach is suitable for both censored and uncensored data.

Combining the aforementioned ingredients, we propose different tests for TE heterogeneity. Our test statistics are suitable functionals of empirical processes whose limiting distribution under the null can be estimated using a multiplicative-type bootstrap. Our proposed tests are of the omnibus type, i.e., they are consistent against any nonparametric fixed alternative. Furthermore, they can detect nonparametric local alternatives converging to the null at the parametric $n^{-1 / 2}$-rate, $n$ being the sample size. To the best of our knowledge, no other nonparametric test for TE heterogeneity share these properties, even when censoring is not an issue.

The closest papers to ours are Abadie (2002), Crump et al. (2008), and Lee (2009). In a context without censoring and covariates, Abadie (2002) proposes tests for the null hypotheses of zero distribution (local) treatment effect and first-order stochastic dominance between treatment and control groups when selection into treatment may be endogenous. Our proposal generalizes Abadie (2002) by accommodating both covariates (and, therefore, treatment effect heterogeneity) and randomly censored outcomes. Crump et al. (2008) propose smoothedbased tests for the null of hypotheses of zero and constant conditional average treatment effects under the unconfoundedness assumption. Our proposal generalizes Crump et al. (2008) by considering tests for treatment effects heterogeneity beyond the conditional mean, and by allowing endogenous treatment allocations and censored outcomes. Finally, Lee (2009) proposes a MannWhitney test for the null hypothesis of zero conditional distribution treatment effect (like $(a)$ above) for randomly censored outcomes. Nonetheless, it is not clear how one can generalize the proposal in Lee (2009) to settings with endogeneity, or how one can use his approach to test other hypotheses related to treatment effect heterogeneity like (b). Delgado and Escanciano (2013), Chang et al. (2015), Hsu (2017), and Lee et al. (2017) propose alternative tests for treatment effect heterogeneity but do not allow for right-censored outcomes.

In summary, we contribute to the literature on different fronts. This paper is the first to 
propose a family of nonparametric tests for TE heterogeneity that $(i)$ can easily accommodate a variety of research designs and (random) censoring and (ii) are able to detect local alternatives converging to null at the parametric rate. In addition, (iii) this paper is one of the first to introduce Kaplan-Meier integrals to the program evaluation literature; see also Sant'Anna (2016) for two-step Kaplan-Meier estimators of different unconditional treatment effect measures.

The remainder of the paper is organized as follows. To gain intuition, we first describe the basic setup in which selection into treatment is exogenous and concentrate on testing the null of zero conditional distribution treatment effect. In Section 3, we derive the asymptotic distribution for the baseline tests and introduce a bootstrap method to approximate their critical values. In Section 4, we present extensions of our basic setup. We consider the null of zero conditional average treatment effect and the null of constant average treatment effect across subpopulations. Furthermore, we show how one can modify the aforementioned tests to accommodate endogenous treatment allocation. A Monte Carlo study in Section 5 investigates the finite sample properties of the tests. In Section 6, we use data from the Illinois Reemployment Bonus Experiment and apply the proposed policy evaluation tools to assess the effect of unemployment insurance bonus on unemployment duration. All mathematical proofs are gathered in the Appendix. Finally, all tests discussed in this article can be implemented via the open-source $\mathrm{R}$ package kmte, which is freely available from GitHub (https://github.com/pedrohcgs/kmte).

Notation: Let $1\{A\}$ be the indicator function, that is, $1\{A\}$ is equal to one if $A$ is true and equal to zero otherwise. When $A$ is a vector, such function is taken coordinatewise. For any generic function $J$, let $J(y-)=\lim _{a \uparrow y} J(a), J\{y\}=J(y)-J(y-)$, and denote the continuous part of $J(\cdot)$ by $J^{c}(\cdot)$. Let $i=\sqrt{-1}$ be the imaginary number. Denote the support of a generic random variable $Z$ by $\mathcal{X}_{Z}$. For a set $\mathcal{W}$, let $l^{\infty}(\mathcal{W})$ be the Banach space of all uniformly bounded real functions on $\mathcal{W}$ equipped with the uniform metric $\|f\|_{\mathcal{W}} \equiv$ $\sup _{z \in \mathcal{W}}|f(z)|$. We use the notation $\|\cdot\|_{\infty}$ to denote the supremum norm. The symbol $\Rightarrow$ denotes weak convergence in $\left(l^{\infty}(\mathcal{W}), \mathcal{W}_{\infty}\right)$ in the sense of J. Hoffmann-J $\phi$ rgensen, where $\mathcal{W}_{\infty}$ denotes the corresponding Borel $\sigma$-algebra, and $\stackrel{p}{\rightarrow}$ denotes convergence in (outer) probability, see e.g. van der Vaart and Wellner (1996). Throughout the paper, all random variables are defined on a common probability space $(\Omega, \mathcal{A}, \mathbb{P})$. 


\section{Testing for zero conditional distribution treatment ef- fect with censored outcomes}

\subsection{Statistical Framework}

We consider a set of individuals flowing into a state of interest, and the time these individuals spend in that state is the outcome of interest, $Y$. Upon inflow, an individual participates in the program or not, i.e., he/she either receives treatment or not. Let $Y(0)$ be the potential outcome if no treatment were received and let $Y(1)$ be the potential outcome if treatment were received. Define $T$ as the treatment indicator, i.e., $T=1$ if the unit is treated and $T=0$ otherwise. The realized outcome is $Y=(1-T) Y(0)+T Y(1)$. The realized outcome, however, is not always observed because of the censoring mechanism. Let $C(0)$ and $C(1)$ be potential censoring random variables under the control and treatment groups, respectively, and $C=(1-T) C(0)+T C(1)$ be the realized censored variable, beyond which $Y$ is not observed. For example, $C$ may be the time from treatment assignment until the end of a follow-up. The observed outcome is $Q=(1-T) Q(0)+T Q(1)$ where $Q(t)=\min (Y(t), C(t))$, $t \in\{0,1\}$. On top of $Q$, the non-censoring indicator $\delta=(1-T) \delta(0)+T \delta(1), \delta(t)=$ $1\{Y(t) \leq C(t)\}, t \in\{0,1\}$, and a vector of pre-treatment variables $\mathbf{X}$ are also observed. We consider $\left\{\left(Q_{i}, \delta_{i}, T_{i}, \mathbf{X}_{i}\right)\right\}_{i=1}^{n}$ as independent and identically distributed (iid) random variables.

Denote the conditional distribution of potential outcomes $Y(0)$ and $Y(1)$ by $F_{Y(0) \mid \mathbf{X}}(y \mid \cdot)$ and $F_{Y(1) \mid \mathbf{X}}(y \mid \cdot)$, respectively, and let the conditional distribution treatment effect be defined as $\Upsilon(y \mid \cdot) \equiv F_{Y(1) \mid \mathbf{X}}(y \mid \cdot)-F_{Y(0) \mid \mathbf{X}}(y \mid \cdot)$. To gain intuition, we first focus on testing the hypothesis that the distribution treatment effect (DTE) is equal to zero for every subpopulation defined by covariates, that is,

$$
H_{0}: \Upsilon(y \mid \mathbf{X})=0 \text { a.s. } \forall y \in \mathcal{W}_{Y},
$$

where $\mathcal{W}_{Y} \subset \mathcal{X}_{Y}$. The alternative hypothesis $H_{1}$ is the negation of $H_{0}$.

A crucial step towards testing (2.1) is to show that $\Upsilon(y \mid \cdot)$ can be identified from the data. To this end, we make the following assumptions.

Assumption 2.1 (i) $(Y(0), Y(1), C(0), C(1)) \Perp T \mid \mathbf{X}$ a.s.; and (ii) for some $\varepsilon>0, \varepsilon \leq$ $\mathbb{P}(T=1 \mid \mathbf{X}) \leq 1-\varepsilon$ a.s..;

Assumption $2.2(i) \quad(Y(0), Y(1)) \Perp \quad(C(0), C(1)) \mid T ;$ and $($ ii $)$ for $t \quad \in \quad\{0,1\}$, $\mathbb{P}(Y(t) \leq C(t) \mid \mathbf{X}, T, Y(t))=\mathbb{P}(Y(t) \leq C(t) \mid T, Y(t))$ a.s. 
We will use the shortcut notation $p_{0}(\mathbf{x}) \equiv \mathbb{P}(T=1 \mid \mathbf{X}=\mathbf{x})$ and refer to $p_{0}(\mathbf{x})$ as the (true) propensity score. Assumption 2.1 is standard in the treatment effects literature. Assumption 2.1(i) states that, conditional on observables, treatment assignment is independent of potential outcomes and censoring. Assumption 2.1(ii) states that there is overlap in the covariate distributions.

In the absence of censoring, Rosenbaum and Rubin (1983) show that Assumption 2.1 suffices to identify different treatment effect measures, in particular $\Upsilon(y \mid \cdot)$. Nonetheless, in our setup censoring introduces an additional identification challenge because the probability of being censored is related to potential outcomes, that is, censoring occurs only if $Y(t)>C(t)$, $t \in\{0,1\}$. Ignoring the censoring problem or analyzing only the uncensored outcomes would, therefore, introduce another source of confounding. To circumvent this problem, Assumption 2.2 imposes additional structure on the censoring mechanism.

Assumption 2.2 states that, conditionally on the treatment status, the potential outcomes are independent of the potential censoring random variables, and that, given the underlying potential outcome $Y(t), t \in\{0,1\}$, and the treatment status $T$, the covariates do not provide any further information on whether censoring will take place. A particular case in which Assumption 2.2 is satisfied is when $C$ is independent of $(Y, \mathbf{X}, T)$, as assumed by Bang and Tsiatis (2000), Anstrom and Tsiatis (2001), Honore et al. (2002), Lee and Lee (2005), Blundell and Powell (2007), among many others. One must bear in mind that Assumption 2.2 is more general than this particular case: it does not impose any restriction on how $(Y(1), Y(0))$ and $(C(1), C(0))$ depend on $T$, it allows some dependence between $C(1), C(0), T$ and $\mathbf{X}$, and allows the occurrence of censoring to depend on $\mathbf{X}$.

In the following, we establish that, given Assumptions 2.1-2.2, a variety of TE measures are identified from $(Q, \delta, T, \mathbf{X})$. In particular, we show that the joint distribution of potential outcome $Y(t)$ and the vector of covariates $\mathbf{X}$, denoted by $F_{Y(t), \mathbf{X}}(y, \mathbf{x})=\mathbb{P}(Y(t) \leq y, \mathbf{X} \leq \mathbf{x})$, $t \in\{0,1\}$ is identified. Once $F_{Y(t), \mathbf{X}}(y, \mathbf{x})$ is identified, $F_{Y(t) \mid \mathbf{X}}(y \mid \cdot)$ can be recovered by taking the appropriate Radon-Nikodym derivative.

We now introduce the multivariate Kaplan-Meier joint distribution, which is the key piece to characterize our Kaplan-Meier integrals. Toward this end, let $H_{Q, \mathbf{X} \mid T}(y, \mathbf{x} \mid t)=\mathbb{P}(Q \leq y, \mathbf{X} \leq$ $\mathbf{x} \mid T=t), H_{Q, \mathbf{X} \mid T}^{1}(y, \mathbf{x} \mid t)=\mathbb{P}(Q \leq y, \mathbf{X} \leq \mathbf{x}, \delta=1 \mid T=t)$ and

$$
\Lambda(y, \mathbf{x} \mid t)=\int_{0}^{y} \frac{H_{Q, \mathbf{X} \mid T}^{1}(d \bar{y}, \mathbf{x} \mid t)}{1-H_{Q, \mathbf{X} \mid T}(\bar{y}-, \infty \mid t)} .
$$


For $t \in\{0,1\}$, let $\tau_{(t)}:=\min \left(\tau_{Y(t)}, \tau_{C(t)}\right)$, where $\tau_{Y(t)}=\inf \{y: \mathbb{P}(Y(t) \leq y)=1\}$, and $\tau_{C(t)}=\inf \{y: \mathbb{P}(C(t) \leq y)=1\}$ are the least upper bound of the support of $Y(t)$ and $C(t)$. For simplicity, assume that $\tau_{C(1)}=\tau_{C(0)}=\tau_{C}, \tau_{Y(1)}=\tau_{Y(0)}=\tau_{Y}$, implying that $\tau_{(1)}=$ $\tau_{(0)}=\tau$. For $t \in\{0,1\}$, denote by $A(t)$ the (possibly empty) set of atoms of the cumulative distribution function of $Q(t)$, and let

$$
F_{Q, \mathbf{X} \mid T}^{k m}(y, \mathbf{x} \mid t)=1-\exp \left(-\Lambda^{c}(y, \mathbf{x} \mid t)\right) \prod_{\bar{y} \leq y}[1-\Lambda(\{\bar{y}\}, \mathbf{x} \mid t)]
$$

where $\Lambda(\{\bar{y}\}, \mathbf{x} \mid t) \equiv \Lambda(\bar{y}, \mathbf{x} \mid t)-\Lambda(\bar{y}-, \mathbf{x} \mid t)$ denotes the jump size (mass) of $\Lambda(y, \mathbf{x} \mid t)$ at $\bar{y}$. In the case where $Q$ is absolutely continuous, $\Lambda(\{\bar{y}\}, \mathbf{x} \mid t) \equiv 0$ for almost all $\bar{y}, \mathbf{x}$ and $t$. In the other extreme case where $Q$ is purely discrete with support points $\left\{y_{1}, y_{2}, \ldots, y_{s}\right\}, \Lambda^{c}(y, \mathbf{x} \mid t) \equiv 0$ for almost all $y, \mathbf{x}$ and $t$, and

$$
\prod_{\bar{y} \leq y}[1-\Lambda(\{\bar{y}\}, \mathbf{x} \mid t)] \equiv\left[1-\Lambda\left(\left\{y_{1}\right\}, \mathbf{x} \mid t\right)\right]^{1\left\{y_{1} \leq y\right\}} \times \cdots \times\left[1-\Lambda\left(\left\{y_{s}\right\}, \mathbf{x} \mid t\right)\right]^{1\left\{y_{s} \leq y\right\}} .
$$

Note that (2.3) allows $Q$ to have both discrete and absolutely continuous components.

Finally, for any measurable function $g(\cdot)$,

$$
\begin{aligned}
\mathbb{E}^{k m}[g(Q, \mathbf{X}, T)] \equiv \int g(T, \mathbf{X}, 1) F_{Q, \mathbf{X} \mid T}^{k m}(d \bar{y}, d \overline{\mathbf{x}} \mid 1) \mathbb{P}(T=1) & \\
& +\int g(Q, \mathbf{X}, 0) F_{Q, \mathbf{X} \mid T}^{k m}(d \bar{y}, d \overline{\mathbf{x}} \mid 0) \mathbb{P}(T=0)
\end{aligned}
$$

Lemma 1 Suppose Assumptions 2.1-2.2 hold. Let $h(\cdot)$ be any measurable function of $(Y, \mathbf{X}, T)$ such that $\mathbb{E}[|h(Y, \mathbf{X}, T)|]<\infty$. Then, for $t \in\{0,1\}$,

$$
\begin{aligned}
& \mathbb{E}^{k m}\left[\frac{1\{T=t\} h(Q, \mathbf{X}, T)}{\mathbb{P}(T=t \mid \mathbf{X})}\right]=\mathbb{E}[h(Y(t), \mathbf{X}, t)1\{Y(t)<\tau\}]+ \\
& 1\{\tau \in A(t)\} \mathbb{E}[1\{Y(t)=\tau\} h(\tau, \mathbf{X}, t)] .
\end{aligned}
$$

Moreover, (2.5) also holds conditional on $\mathbf{X}$.

Lemma 1 is based on Sant'Anna (2016), and extends Rosenbaum and Rubin (1983) identification results to setups with censored outcomes. It relies on replacing $F_{Y, \mathbf{X} \mid T}$ by the multivariate Kaplan-Meier $F_{Q, \mathbf{X} \mid T}^{k m}$ in (2.4). Note that $F_{Q, \mathbf{X} \mid T}^{k m}$ only depends on $(Q, \delta, T, \mathbf{X})$ and, therefore, is self-adjusted to the censoring problem. Furthermore, in the absence of censoring, $F_{Q, \mathbf{X} \mid T}^{k m}=F_{Y, \mathbf{X} \mid T}$ a.s. (Shorack and Wellner, 1986, Proposition 1, pg. 301), implying that in such a case, our identification result reduces to those of Rosenbaum and Rubin (1983). On the other hand, when the outcome of interest is subject to random censoring but one ignores this 
feature and treats $Q$ as $Y$, the "standard" IPW estimand can be expressed as

$$
\begin{aligned}
\mathbb{E}\left[\frac{1\{T=t\} h(Q, X, T)}{\mathbb{P}(T=t \mid X)}\right]=\mathbb{E}[h(Y(t), & X, t) \mid Y(t) \leq C(t)] \mathbb{P}(Y(t) \leq C(t)) \\
& +\mathbb{E}[h(C(t), X, t) \mid Y(t)>C(t)] \mathbb{P}(Y(t)>C(t)) .
\end{aligned}
$$

In other words, if one ignores the censoring problem, one would only identify a weighted average of functionals of $(i)(Y(t), X, t)$ for the uncensored subpopulation $(Y(t) \leq C(t)$ a.s. $)$ and (ii) $(C(t), X, t)$ for the censored subpopulation $(Y(t)>C(t)$ a.s. $)$. Such weighted average representations do not have any clear causal interpretation as these subpopulations (being censored or not) are directly related to the potential outcomes. We bypass the additional source of confounding introduced by the censoring problems by using the multivariate Kaplan-Meier distribution $F_{Q, \mathbf{X} \mid T}^{k m}$ as an integrating measure.

It is important to remark that, because of the censoring problem, nonparametric identification of statistical characteristics that depend on the entire support of $Y(t)$ such as $\mathbb{E}[Y(t)]$, $t \in\{0,1\}$, crucially depends on the local structure of the distribution of $Y(t)$ and $C(t)$ at their endpoint. If $\tau_{Y}<\tau_{C}$, identification is guaranteed for any (well defined) functional of interest; if $\tau_{Y}>\tau_{C}$, only restricted moments can be identified; and if $\tau_{C}=\tau_{Y}$, identification is guaranteed unless

$$
F_{Y(t), \mathbf{X}}(\{\tau\}, \mathbf{x})>0 \text { and } \mathbb{P}(C(t)<\tau)=1,
$$

where $F_{Y(t), \mathbf{X}}(\{\tau\}, \mathbf{x}) \equiv F_{Y(t), \mathbf{X}}(\tau, \mathbf{x})-F_{Y(t), \mathbf{X}}(\tau-, \mathbf{x})$. In particular, whenever $Y(t)$ is continuous, $\mathbb{E}[h(Y(t), \mathbf{X}, t)]$ is nonparametrically identified if $\tau_{Y} \leq \tau_{C}$, otherwise one can only identify $\mathbb{E}[h(Y(t), \mathbf{X}, t) 1\{Y(t) \leq \tau\}]$. This is intuitive because relevant information about $F_{Y(t), \mathbf{X}}$ on $\left(\tau_{C}, \tau_{Y}\right]$ will always be cut off as a result of the censoring. Such information cannot be recovered unless one is willing to rely on additional parametric/shape restrictions. As indicated by (2.5) and (2.6), the case of discrete potential outcomes and potential censoring random variables is more delicate: if the the underlying distribution of $Y(t)$ has a positive mass point at $\tau$ but the $\mathbb{P}(C(t) \geq \tau)=0$, no information about $Y(t)$ at $\tau$ can be recovered from $(Q, \mathbf{X}, T)^{1}$.

1 Under Assumptions 2.1 and 2.2, and with no covariates, it is easy to see the lack of identification at the boundary of the support may appear when $H$ is not absolutely continuous. In light of (2.2) and (2.3), if $F_{Y(t)}$ has a mass point at $\tau, F_{Y(t)}(\{\tau\})=F_{Y(t)}(\tau)-F_{Y(t)}(\tau-)$, one would naturally hope to identify if by using integrals of $H_{t, 1}(\tau)-H_{t, 1}(\tau-)=-\left(\left(1-H_{t, 1}(\tau)\right)-1-H_{t, 1}(\tau-)\right), t=0,1$. From the definition of $\tau$, we have that, for $t=0,1,0=1-H_{Q \mid T}(\tau \mid t)=\left(1-F_{Y(t)}(\tau)\right)\left(1-G_{C(t)}(\tau)\right)$. When $F_{Y(t)}(\tau-)<1$ but $G_{C(t)}(\tau-)=1$, we have that $\tau_{(t)} \notin A(t)$, and $1-H_{Q \mid T}(\tau-\mid t)=\left(1-F_{Y(t)}(\tau-)\right) \times 0=0$. Thus, $H_{t, 1}(\tau)-H_{t, 1}(\tau-)=0$, implying that if $Y(t)$ has a mass point at $\tau \notin A(t)$, one cannot recover information about it from $(Q, T)$. On the other hand, if $F_{Y(t)}(\tau-)<1$ but $G_{C(t)}(\tau-)<1$, then the identification follows naturally as one would be able to observe $Q=\tau$ for those with $\delta=1$. 
As a direct consequence, in such a case, only restricted moments of potential outcomes can be nonparametrically point identified. Indeed, the second term in (2.5) emphasizes this feature. In order to ease notation and avoid repetition of arguments, in the rest of the paper, we rule out (2.6).

One should bear in mind that although nonparametric identification of general statistical characteristics is not always guaranteed, Lemma 1 is still very powerful. For instance, applying Lemma 1 with $h(Y, \mathbf{X}, T)=1\{Y \leq y\} 1\{\mathbf{X} \leq \mathbf{x}\}$, we get that $F_{Y(t), \mathbf{X}}(y, \mathbf{x})$ and $F_{Y(t) \mid \mathbf{X}}(y \mid \mathbf{x})$ are identified for $(y, \mathbf{x}) \in[-\infty, \tau] \times \mathcal{X}_{X}$. This is in contrast to the results of Frangakis and Rubin (1999), Anstrom and Tsiatis (2001), and Frandsen (2015), who restrict the analysis to $y \in$ $[-\infty, \bar{\tau}]$, with $\bar{\tau}<\tau$. In practice, given that there is no general rule on how to appropriately choose $\bar{\tau}$, an ad hoc choice of "small" $\bar{\tau}$ can lead to undesirable loss of information. The results in Lemma 1 completely avoid such a drawback ${ }^{2}$.

\subsection{Characterization of the null hypothesis}

From Lemma 1, we have that, for $y \in[-\infty, \tau]$, the conditional DTE $\Upsilon(y \mid \cdot)$ is identified from the data and, therefore, we are able to characterize the null hypothesis (2.1) in terms of observables.

One approach to constructing tests for (2.1) is to combine Lemma 1 with smoothing techniques, estimate the conditional DTE $\Upsilon(y \mid \cdot)$, and then compare how close $\Upsilon(y \mid \cdot)$ is to zero. The main drawback of this strategy is that, when the dimension of covariates $\mathbf{X}$ is moderate as is commonly the case in policy evaluation, tests based on this local approach suffers from the "curse of dimensionality"; see e.g. Fan and Li (1996) for related tests in a different context. In the next Lemma, we show that, by exploiting alternative characterizations of (2.1), one can avoid estimating $\Upsilon(y \mid \cdot)$, alleviating the drawback associated with the local approach described above. To do so, we rely on the "integrated moment approach" used in the goodness-of-fit test literature, see e.g. Bierens (1982), Stute (1997), Escanciano (2006), among others.

\section{Lemma 2 Suppose Assumptions 2.1-2.2 hold. Assume that the parametric family}

2 The aforementioned papers rely on inverse probability weighting techniques to achieve identification. As such, when $y$ approaches $\tau$, the probability of censoring approaches one, implying that the associated inverse probability of censoring weights approach infinity. Therefore, it is not straightforward to see how one can adapt the identification arguments used in these papers to the case where $\tau=\infty$, for example. Finally, we note that these boundary problems can become even more perverse when one turns attention to estimation. 
$\mathcal{F}=\left\{w(\mathbf{X}, \mathbf{x}): \mathbf{x} \in \Pi \subset[-\infty, \infty]^{k}\right\}$ satisfy Assumption A.1 stated in Appendix A. Then,

$$
\Upsilon(y \mid \mathbf{X})=0 \text { a.s. } \forall y \in[-\infty, \tau] \Leftrightarrow I_{w}(y, \mathbf{x})=0 \text { a.e. in }[-\infty, \tau] \times \Pi
$$

where $\Pi$ is a properly chosen space that may depend on the choice of $w, I_{w}(y, \mathbf{x})=I_{w}^{1}(y, \mathbf{x})-$ $I_{w}^{0}(y, \mathbf{x})$, and for $t \in\{0,1\}$,

$$
I_{w}^{t}(y, \mathbf{x}) \equiv \mathbb{E}^{k m}\left[\frac{1\{T=t\} 1\{Q \leq y\}}{\mathbb{P}(T=t \mid \mathbf{X})} w(\mathbf{X}, \mathbf{x})\right],
$$

Lemma 2 adapts Lemma 1 of Escanciano (2006) to the present context. Examples of parametric families $w(\cdot, \mathbf{x})$ such that the equivalence $(2.7)$ holds are the exponential function $w(\mathbf{X}, \mathbf{x})=\exp \left(i \mathbf{x}^{\prime} \mathbf{X}\right)$ with $\mathbf{x} \in \mathbb{R}^{k}$, as in Bierens (1982), and the indicator function $w(\mathbf{X}, \mathbf{x})=1\{\mathbf{X} \leq \mathbf{x}\}$ with $\mathbf{x} \in \mathcal{X}_{X}$, as in Stute (1997); for alternative weight functions, see e.g. Stinchcombe and White (1998).

From (2.7), it is indeed clear that in order to test for (2.1), it suffices to estimate $I_{w}(y, \mathbf{x})$ and check if it is "sufficiently close to zero" for all values of $(y, \mathbf{x}) \in \mathcal{W} \subseteq[-\infty, \tau] \times \Pi$. Of course, the notion of "sufficiently close" depends on $(i)$ the norm one uses, say, the $L_{2}$-norm, which leads to a Cramér-von Mises-type test, or the sup-norm, which leads to a KolmogorovSmirnov-type test; and $(i i)$ the sampling distribution of the estimator of $I_{w}(y, \mathbf{x})$. In the next section, we discuss in detail how one can actually estimate $I_{w}(y, \mathbf{x})$, and how one can use these estimators to form test-statistics for (2.1).

\section{$2.3 \quad$ Test statistic}

The characterization of the null hypothesis in (2.7) suggests using functionals of an estimator of $I_{w}(\cdot, \cdot)$ as test statistics. Therefore, we must first estimate $I_{w}(\cdot, \cdot)$ using the sample $\left\{\left(Q_{i}, \delta_{i}, T_{i}, \mathbf{X}_{i}\right)\right\}_{i=1}^{n}$. From Lemma 1 and the Total Law of Probability, we have that, for $(y, \mathbf{x}) \in[-\infty, \tau] \times \Pi, t \in\{0,1\}$,

$$
I_{w}^{t}(y, \mathbf{x})=\mathbb{P}(T=t) \int \frac{1\{\bar{y} \leq y\} w(\overline{\mathbf{x}}, \mathbf{x})}{\mathbb{P}(T=t \mid \mathbf{X}=\overline{\mathbf{x}})} F_{Q, \mathbf{X} \mid T}^{k m}(d \bar{y}, d \overline{\mathbf{x}} \mid t) .
$$

Thus, to estimate $I_{w}(\cdot, \cdot)$, we have to estimate $\mathbb{P}(T=t \mid \mathbf{X}), F_{Q, \mathbf{X} \mid T}^{k m}(y, \mathbf{x} \mid t)$ and $\mathbb{P}(T=t), t \in$ $\{0,1\}$.

The task of estimating the propensity score $p_{0}(\cdot)$ is relatively standard. For instance, when the data comes from a randomized experiment, $p_{0}(\cdot)$ can be estimated by $n^{-1} \sum_{i=1}^{n} T_{i}$. Alternatively, when the treatment allocation depends on observable characteristics, one can nonparametrically estimate $p_{0}(\cdot)$ using the Series Logit Estimator (SLE) proposed by Hirano et al. 
(2003). To define the SLE, let $\boldsymbol{\lambda}=\left(\lambda_{1}, \ldots, \lambda_{r}\right)^{\prime}$ be a $r$-dimensional vector of non-negative integers with norm $|\boldsymbol{\lambda}|=\sum_{j=1}^{r} \lambda_{j}$. Let $\{\boldsymbol{\lambda}(l)\}_{l=1}^{\infty}$ be a sequence including all distinct multi-indices $\boldsymbol{\lambda}$ such that $|\boldsymbol{\lambda}(l)|$ is non-decreasing in $l$ and let $\mathbf{x}^{\lambda}=\prod_{j=1}^{r} \mathbf{x}_{j}^{\lambda_{j}}$. For any integer $L$, define $\mathbf{R}^{L}(\mathbf{x})=\left(\mathbf{x}^{\lambda(1)}, \ldots, \mathbf{x}^{\lambda(L)}\right)^{\prime}$ as a vector of power functions. Let $\mathcal{L}(a)=\exp (a) /(1+\exp (a))$ be the logistic $C D F$. The SLE for $p_{0}(\mathbf{x})$ is defined as $\hat{p}_{n}(\mathbf{x})=\mathcal{L}\left(\mathbf{R}^{L}(x)^{\prime} \hat{\boldsymbol{\pi}}_{L}\right)$, where

$$
\hat{\boldsymbol{\pi}}_{L}=\arg \max _{\boldsymbol{\pi}_{L}} \frac{1}{n} \sum_{i=1}^{n} T_{i} \log \left(\mathcal{L}\left(\mathbf{R}^{L}\left(\boldsymbol{X}_{i}\right)^{\prime} \boldsymbol{\pi}_{L}\right)\right)+\left(1-T_{i}\right) \log \left(1-\mathcal{L}\left(\mathbf{R}^{L}\left(\boldsymbol{X}_{i}\right)^{\prime} \boldsymbol{\pi}_{L}\right)\right) .
$$

We write $\hat{\mathbb{P}}_{n}(T=1 \mid \mathbf{X}=\mathbf{x})=\hat{p}_{n}(\mathbf{x})$ and $\hat{\mathbb{P}}_{n}(T=0 \mid \mathbf{X}=\mathbf{x})=1-\hat{p}_{n}(\mathbf{x})$.

Next, we move to the most challenging step: estimating $F_{Q, \mathbf{X} \mid T}^{k m}(y, \mathbf{x} \mid t)$. Note that, as a result of the binary nature of $T$, we have to estimate two distribution functions: $F_{Q, \mathbf{X} \mid T}^{k m}(y, \mathbf{x} \mid 1)$, and $F_{Q, \mathbf{X} \mid T}^{k m}(y, \mathbf{x} \mid 0)$. To this end, we divide the data $\left\{\left(Q_{i}, \delta_{i}, T_{i}, \mathbf{X}_{i}\right)\right\}_{i=1}^{n}$ into two sub-samples given by different values of treatment status $T ;\left\{\left(Q_{i}, \delta_{i}, \mathbf{X}_{i}\right)\right\}_{i=1}^{n_{1}}$ are those observations with $T_{i}=1\left(n_{1}=\sum_{i} T_{i}\right)$; and $\left\{\left(Q_{i}, \delta_{i}, \mathbf{X}_{i}\right)\right\}_{i=1}^{n_{0}}$ are those observations with $T_{i}=0\left(n_{0}=\sum_{i}\left(1-T_{i}\right)\right)$. Then, the task of estimating $F_{Q, \mathbf{X} \mid T}^{k m}(y, \mathbf{x} \mid t)$ is reduced to estimating (2.2) and plugging it into (2.3). We estimate $\Lambda(y, \mathbf{x} \mid t)$ by replacing $H_{Q, \mathbf{X} \mid T}^{1}(y, \mathbf{x} \mid t)$ and $H_{Q, \mathbf{X} \mid T}(y-, \infty \mid t)$ with their empirical analogues, leading to the estimator

$$
\hat{\Lambda}_{n}(y, \mathbf{x} \mid t)=\sum_{i=1}^{n_{t}} \frac{1\left\{Q_{i: n_{t}} \leq y\right\} 1\left\{\mathbf{X}_{\left[i: n_{t}\right]} \leq \mathbf{x}\right\} \delta_{\left[i: n_{t}\right]}}{n_{t}-i+1}
$$

where $Q_{1: n_{t}} \leq \cdots \leq Q_{n_{t}: n_{t}}$ are the ordered $Q$-values in the sub-sample with $\{T=t\}$, and $\mathbf{X}_{\left[i: n_{t}\right]}$ and $\delta_{\left[i: n_{t}\right]}$ are the concomitants of the $i-t h$ order statistic, that is, the $\mathbf{X}$ and $\delta$ paired with $Q_{i: n_{t}}$. Here, ties within outcomes of interest or censoring random variables are ordered arbitrarily, and ties among $Y$ and $C$ are treated as if the former precedes the latter. By plugging $\hat{\Lambda}_{n}(y, \mathbf{x} \mid t)$ into (2.3), and noticing that $\hat{\Lambda}_{n}(y, \mathbf{x} \mid t)$ is a step function, we have that a natural estimator for $F_{Q, \mathbf{X} \mid T}^{k m}(y, \mathbf{x} \mid t)$ is

$$
\hat{F}_{Q, \mathbf{X} \mid T, n}^{k m}(y, \mathbf{x} \mid t)=1-\prod_{\bar{y} \leq y}\left[1-\hat{\Lambda}_{n}(\{\bar{y}\}, \mathbf{x} \mid t)\right]
$$

which is the multivariate extension of the time-honored Kaplan and Meier (1958) product limit estimator proposed by Stute (1993). As $\hat{F}_{Q, \mathbf{X} \mid T, n}^{k m}(y, \mathbf{x} \mid t)$ is a step function, it can be seen from (2.10) and (2.11) that

$$
\hat{F}_{Q, \mathbf{X} \mid T, n}^{k m}(y, \mathbf{x} \mid t)=\sum_{i=1}^{n_{t}} W_{i n_{t}} 1\left\{Q_{i: n_{t}} \leq y\right\} 1\left\{\mathbf{X}_{\left[i: n_{t}\right]} \leq \mathbf{x}\right\},
$$

where, for $1 \leq i \leq n_{t}$,

$$
W_{i n_{t}}=\frac{\delta_{\left[i: n_{t}\right]}}{n_{t}-i+1} \prod_{j=1}^{i-1}\left[\frac{n_{t}-j}{n_{t}-j+1}\right]^{\delta_{\left[j: n_{t}\right]}}
$$


is the Kaplan-Meier weight attached to $Q_{i: n_{t}}, t \in\{0,1\}$.

Finally, given the discrete nature of $T$, we can nonparametrically estimate $\mathbb{P}(T=t)$ by its relative frequency $n_{t} / n$. Putting all these pieces together, we have that

$$
\hat{I}_{w, n}(y, \mathbf{x})=\hat{I}_{w, n}^{1}(y, \mathbf{x})-\hat{I}_{w, n}^{0}(y, \mathbf{x}),
$$

where, for $t \in\{0,1\}$,

$$
\hat{I}_{w, n}^{t}(y, \mathbf{x})=\frac{n_{t}}{n} \sum_{i=1}^{n_{t}} W_{i n_{t}} \frac{1\left\{Q_{i: n_{t}} \leq y\right\} w\left(\mathbf{X}_{\left[i: n_{t}\right]}, \mathbf{x}\right)}{\hat{\mathbb{P}}_{n}\left(T=t \mid \mathbf{X}_{\left[i: n_{t}\right]}\right)} .
$$

In the absence of censoring, $W_{i n_{t}}=n_{t}^{-1}$ a.s., and (2.14) is reduced to the empirical analogue of (2.8). Thus, it is evident that our proposal is suitable for both censored and uncensored outcomes.

With $\hat{I}_{w, n}(y, \mathbf{x})$ at hand, testing the null hypothesis (2.1) is relatively straightforward: for a given weighting function $w(\cdot, \mathbf{x})$, we just need to compare how close $\sqrt{n} \hat{I}_{w, n}(y, \mathbf{x})$ is to zero. We consider the usual sup and $L_{2}$ norms, with the indicator weighting function $w(\mathbf{X}, \mathbf{x})=1\{\mathbf{X} \leq \mathbf{x}\}$, leading to the Kolmogorov-Smirnov $(K S)$ and Cramér-von Mises $(C v M)$ test statistics

$$
\begin{aligned}
K S_{n} & =\sqrt{n} \sup _{(y, \mathbf{x}) \in \mathcal{W}}\left|\hat{I}_{1, n}(y, \mathbf{x})\right| \\
C v M_{n} & =n \int_{\mathcal{W}}\left|\hat{I}_{1, n}(y, \mathbf{x})\right|^{2} \hat{H}_{n}(d y, d \mathbf{x}),
\end{aligned}
$$

respectively, where $\hat{I}_{1, n}(y, \mathbf{x})$ is defined as $\hat{I}_{w, n}(y, \mathbf{x})$ with $w(\mathbf{X}, \mathbf{x})=1\{\mathbf{X} \leq \mathbf{x}\}, \hat{H}_{n}(y, \mathbf{x})$ denotes the sample analog of $H(y, \mathbf{x})=\mathbb{P}(Q \leq y, \mathbf{X} \leq \mathbf{x})$, and $\mathcal{W}=[-\infty, \tau] \times \mathcal{X}_{X}$. Obviously, different test statistics could be developed by applying other distances, or choosing alternative weighting functions $w$, but for ease of exposition, we concentrate of $K S_{n}$ and $C v M_{n}$. To avoid cumbersome notation, in the rest of the article, we consider $\mathcal{W}=[-\infty, \tau] \times \mathcal{X}_{X}$.

Remark 1 In some circumstances, researchers may be interested in assessing if the DTE is equal to zero for every subpopulation defined by a subset of $\mathbf{X}$, say $\mathbf{X}_{1}$. This is particularly attractive when the dimension of $\mathbf{X}$ is moderate so one can plausibly rely on the unconfoundedness assumption, but the covariates of interest for analyzing heterogeneity are of much lower dimension; see e.g. Abrevaya et al. (2015) and Lee et al. (2017). In this situation, instead of testing for (2.1), the goal would be testing the null

$$
H_{0}^{s u b}: F_{Y(1) \mid \mathbf{X}_{1}}(y \mid \cdot)-F_{Y(0) \mid \mathbf{X}_{1}}(y \mid \cdot)=0 \text { a.s. } \forall y \in[-\infty, \tau] .
$$

Note that by setting $w(\mathbf{X}, \mathbf{x})=1\left(\mathbf{X}_{1} \leq \mathbf{x}_{1}\right)$, or more generally, $w(\mathbf{X}, \mathbf{x})=w\left(\mathbf{X}_{1}, \mathbf{x}_{1}\right)$, our 
tests can cover this type of hypothesis in a rather straightforward manner.

Remark 2 In some applications, researchers may be comfortable assuming that the treatment effect cannot be "negative" for any subgroup. In such cases, one may want to test

$$
H_{0}: \Upsilon(y \mid \mathbf{X})=0 \text { a.s. } \forall y \in[-\infty, \tau]
$$

against the one-sided alternative hypothesis

$$
H_{1}: \mathbb{P}(\Upsilon(y \mid \mathbf{X})>0)>0 \text { for some } y \in[-\infty, \tau]
$$

using the test statistic

$$
K S_{n}^{\text {one }}=\sup _{(y, \mathbf{x}) \in \mathcal{W}} \sqrt{n} \hat{I}_{n}(t, \mathbf{x}) .
$$

The statistical analysis of one-sided test based on (2.17) follows similar arguments as the twosided test based on (2.15).

We note that in settings without censoring, one can consider more general tests for conditional stochastic dominance that do not rely on external information about the direction of the (distributional) treatment effect, see e.g. Andrews and Shi $(2013,2017)$ and Hsu (2017). Extending the results in Andrews and Shi (2013, 2017) and Hsu (2017) to allow for random censoring, however, involves very technical challenges related to establishing a uniform in DGP representation for Kaplan-Meier processes and is, therefore, beyond the scope of this paper; see the Conclusion Section for further details.

\section{Asymptotic Theory}

\subsection{Asymptotic linear representation}

We now discuss the asymptotic theory for our test statistics using the following notation. For $t \in\{0,1\}$, let $H_{t}(y)=\mathbb{P}(Q \leq y, T=t), H_{t, 0}(y)=\mathbb{P}(Q \leq y, \delta=0, T=t)$, and $H_{t, 11}(y, x)=$ $\mathbb{P}(Q \leq y, \mathbf{X} \leq \mathbf{x}, \delta=1, T=t)$. Define

$$
\gamma_{t, 0}(\bar{y})=\exp \left\{\int_{0}^{\bar{y}-} \frac{H_{t, 0}(d \bar{w})}{1-H_{t}(\bar{w})}\right\} .
$$

Let

$$
\gamma_{t, 1}(\bar{y} ; y, \mathbf{x})=\frac{1}{1-H_{t}(\bar{y})} \int 1\{\bar{y}<\bar{w}\} \xi_{t}(\bar{w}, \overline{\mathbf{x}}, t ; y, \mathbf{x}) \gamma_{t, 0}(\bar{w}) H_{t, 11}(d \bar{w}, d \overline{\mathbf{x}})
$$

and

$$
\gamma_{t, 2}(\bar{y} ; y, \mathbf{x})=\iint \frac{1\{\bar{v}<\bar{y}, \bar{v}<\bar{w}\} \xi_{t}(\bar{w}, \overline{\mathbf{x}}, t ; y, \mathbf{x})}{\left[1-H_{t}(\bar{v})\right]^{2}} \gamma_{t, 0}(\bar{w}) H_{t, 0}(d \bar{v}) H_{t, 11}(d \bar{w}, d \overline{\mathbf{x}}),
$$


where

$$
\begin{aligned}
\xi_{1}(Q, \mathbf{X}, T ; y, \mathbf{x}) & =\frac{T 1\{Q \leq y\} 1\{\mathbf{X} \leq \mathbf{x}\}}{p_{0}(\mathbf{X})} \\
\xi_{0}(Q, \mathbf{X}, T ; y, \mathbf{x}) & =\frac{(1-T) 1\{Q \leq y\} 1\{\mathbf{X} \leq \mathbf{x}\}}{1-p_{0}(\mathbf{X})}
\end{aligned}
$$

Put

$$
\eta_{t, i}(y, \mathbf{x})=\xi_{t}\left(Q_{i}, \mathbf{X}_{i}, T_{i} ; y, \mathbf{x}\right) \gamma_{t, 0}\left(Q_{i}\right) \delta_{i}+\gamma_{t, 1}\left(Q_{i} ; y, \mathbf{x}\right)\left(1-\delta_{i}\right)-\gamma_{t, 2}\left(Q_{i} ; y, \mathbf{x}\right)
$$

Some remarks are necessary. First, (3.6) relies only on the "known" functions $\xi_{t}, t \in$ $\{0,1\}$. Then, as discussed in Stute $(1995,1996)$, the first term of $\eta_{t, i}(y, \mathbf{x})$ has expectation $\mathbb{E}\left[\xi_{t}(Q, \mathbf{X}, T ; y, \mathbf{x})\right]$. The second and third terms have identical expectations and appear because of the censoring. As is expected and desired, in the absence of censoring, $\gamma_{t, 0}(\cdot)=1$ a.s., and $\gamma_{t, 1}(\cdot)=\gamma_{t, 2}(\cdot)=0$ a.s..

Given that $\hat{I}_{1, n}(\cdot, \cdot)$ is the difference of two empirical KM integrals, define

$$
\eta_{i}(y, \mathbf{x})=\eta_{1, i}(y, \mathbf{x})-\eta_{0, i}(y, \mathbf{x})
$$

the difference of (3.6) between the treated and control group.

To discuss the estimation effect coming from not knowing $p_{0}(\cdot)$ in the KM-integrals, let

$$
\alpha_{1}(\mathbf{X} ; y, \mathbf{x})=-\frac{F_{Y(1) \mid \mathbf{X}}(y \mid \mathbf{X}) 1\{\mathbf{X} \leq \mathbf{x}\}}{p_{0}(\mathbf{X})}, \alpha_{0}(\mathbf{X} ; y, \mathbf{x})=\frac{F_{Y(0) \mid \mathbf{X}}(y \mid \mathbf{X}) 1\{\mathbf{X} \leq \mathbf{x}\}}{1-p_{0}(\mathbf{X})}
$$

Notice that $\alpha_{1}(\cdot ; y, \mathbf{x})$ and $\alpha_{0}(\cdot ; y, \mathbf{x})$ are nothing more than the conditional expectation of the (functional) derivative of $(3.4)$ and (3.5) with respect to $p_{0}(\cdot)$, respectively. Similarly to (3.7), define

$$
\alpha(\mathbf{X} ; y, \mathbf{x})=\alpha_{1}(\mathbf{X} ; y, x)-\alpha_{0}(\mathbf{X} ; y, \mathbf{x})
$$

In order to present our asymptotic results, we need to assume some additional regularity conditions related to the estimation of the propensity score $p_{0}(\cdot)$, and some integrability conditions to guarantee that the variance of our test statistics is finite and that the censoring effects do not dominate in the right tail. These technical assumptions are stated in Appendix A.

Lemma 3 Under Assumptions 2.1-2.2 and Assumptions A.2-A.6 stated in Appendix A, we have

$\sqrt{n}\left(\hat{I}_{1, n}(y, \mathbf{x})-I_{1}(y, \mathbf{x})\right)=\frac{1}{\sqrt{n}} \sum_{i=1}^{n}\left\{\left[\eta_{i}(y, \mathbf{x})-I_{1}(y, \mathbf{x})\right]+\alpha\left(\mathbf{X}_{i} ; y, \mathbf{x}\right)\left(T_{i}-p_{0}\left(\mathbf{X}_{i}\right)\right)\right\}+o_{\mathbb{P}}(1)$ uniformly in $(y, \mathbf{x}) \in \mathcal{W}$. 


\subsection{Asymptotic null distribution}

Using the uniform representation from Lemma 3, we next establish the weak convergence of the processes $\sqrt{n} \hat{I}_{1, n}(y, \mathbf{x})$ under the null hypothesis

$$
H_{0}: \Upsilon(y \mid \mathbf{X})=0 \text { a.s. } \forall y \in[-\infty, \tau] .
$$

Theorem 1 Under the null hypothesis (3.10), Assumptions 2.1-2.2 and Assumptions A.2-A.6 stated in Appendix A, we have

$$
\sqrt{n} \hat{I}_{1, n}(y, \mathbf{x}) \Rightarrow C_{\infty}(y, \mathbf{x})
$$

where $C_{\infty}$ is Gaussian process with zero mean and covariance function

$$
V\left(\left(y_{1}, \mathbf{x}_{1}\right),\left(y_{2}, \mathbf{x}_{2}\right)\right)=\mathbb{E}\left[\psi\left(y_{1}, \mathbf{x}_{1}\right) \psi\left(y_{2}, \mathbf{x}_{2}\right)\right]
$$

with $\psi(y, \mathbf{x})=\eta(y, \mathbf{x})+\alpha(\mathbf{X} ; y, \mathbf{x})(T-p(\mathbf{X}))$.

Now, we can apply the continuous mapping theorem to characterize the limiting null distribution of our test statistics using the sup and $L_{2}$ distances.

Corollary 1 Under the null hypothesis (3.10) and the Assumptions of Theorem 1,

$$
\begin{aligned}
& K S_{n} \stackrel{d}{\rightarrow} \sup _{(y, \mathbf{x}) \in \mathcal{W}}\left|C_{\infty}(y, \mathbf{x})\right| \\
& C v M_{n} \stackrel{d}{\rightarrow} \int_{\mathcal{W}}\left|C_{\infty}(y, \mathbf{x})\right|^{2} H(d y, d \mathbf{x}) .
\end{aligned}
$$

Let $T_{n}$ be a generic notation for $K S_{n}$ and $C v M_{n}$. From Corollary 1, it follows immediately that

$$
\lim _{n \rightarrow \infty} \mathbb{P}\left\{T_{n}>c_{\alpha}^{T}\right\}=\alpha
$$

where $c_{\alpha}^{T}=\inf \left\{c \in[0, \infty): \lim _{n \rightarrow \infty} \mathbb{P}\left\{T_{n}>c\right\}=\alpha\right\}$.

\subsection{Asymptotic power against fixed and local alternatives}

Now we analyze the asymptotic properties of our tests under the fixed alternative $H_{1}$. Under $H_{1}, \mathbb{P}(\Upsilon(y \mid \mathbf{X})=0)<1$ for some $y \in[-\infty, \tau]$, implying that $I_{1}(y, \mathbf{x}) \neq 0$ for some $(y, \mathbf{x}) \in \mathcal{W}$. Therefore, our test statistics $K S_{n}$ and $C v M_{n}$ diverge to infinity. Given that the critical values are bounded, it follows that our tests are consistent. We formalize this result in the next theorem.

Theorem 2 Under the fixed alternative hypothesis

$$
H_{1}: \mathbb{P}(\Upsilon(y \mid \mathbf{X})=0)<1 \text { for some } y \in[-\infty, \tau]
$$


and the Assumptions of Theorem 1,

$$
\begin{array}{r}
\lim _{n \rightarrow \infty} \mathbb{P}\left\{K S_{n}>c_{\alpha}^{K S}\right\}=1, \\
\lim _{n \rightarrow \infty} \mathbb{P}\left\{C v M_{n}>c_{\alpha}^{C v M}\right\}=1 .
\end{array}
$$

Given that our test statistics diverge to infinity under fixed alternatives, it is desirable studying the asymptotic power of these tests under local alternatives. To this end, we study the asymptotic behavior of $\hat{I}_{1, n}(y, \mathbf{x})$ under alternative hypotheses converging to the null at the parametric rate $n^{-1 / 2}$.

Consider the following class of local alternatives:

$$
H_{1, n}: \Upsilon(y \mid \mathbf{X})=\frac{h(y, \mathbf{X})}{\sqrt{n}} \text { a.s. } \forall y \in[-\infty, \tau] .
$$

In the sequel, we need that (3.12) satisfies the following regularity condition.

Assumption 3.1 (a) $h(\cdot, \cdot)$ is an F-integrable function;

(b) the set $h_{n} \equiv\left[(y, \mathbf{x}) \in \mathcal{W}: n^{-1 / 2} h(y, \mathbf{x}) \neq 0\right]$ has positive Lebesgue measure.

Theorem 3 Under local alternatives (3.12), Assumptions 2.1-2.2, 3.1 and Assumptions A.2A.6 stated in Appendix A,

$$
\sqrt{n} \hat{I}_{1, n}(y, \mathbf{x}) \Rightarrow C_{\infty}(y, \mathbf{x})+R(y, \mathbf{x})
$$

where $C_{\infty}$ is the process defined in Theorem 1 and $R(y, \mathbf{x}) \equiv \mathbb{E}[h(y, \mathbf{X}) 1\{\mathbf{X} \leq \mathbf{x}\}]$

From the above theorem and straightforward application of the continuous mapping theorem, we see that our test statistics, under local alternatives of the form of (3.12), converge to a different distribution because of the presence of a deterministic shift function $R$. This additional term guarantees the good local power property of our tests.

\subsection{Estimation of critical values}

From the above theorems, we see that the asymptotic distribution of $\sqrt{n} \hat{I}_{1, n}(\cdot, \cdot)$ depends on the underlying data generating process and standardization is complicated. To overcome this problem, we propose computing critical values with the assistance of a multiplier bootstrap. The proposed procedure has good theoretical and empirical properties, is straightforward to verify its asymptotic validity, is computationally easy to implement and does not require computing new parameter estimates at each bootstrap replication. 
To implement the bootstrap, we need nonparametric estimators for all the terms in the asymptotic linear representation of Lemma 3 , namely the propensity score $p_{0}(\cdot), \eta(y, \mathbf{x})$ as in (3.7), and $\alpha(\cdot ; y, \mathbf{x})$ as in (3.9).

As already discussed, we estimate $p_{0}(\cdot)$ using the SLE of Hirano et al. (2003). In order to estimate $\eta(y, \mathbf{x})$, we notice that after plugging in $\hat{p}_{n}(\cdot)$, each $\gamma$ only depends on $H$-functions and is, therefore, estimable by just replacing the $H$-terms by their empirical counterparts. Then, we estimate $\eta(y, \mathbf{x})$ by its empirical analogue

$$
\hat{\eta}_{n}(y, \mathbf{x})=\hat{\eta}_{1, n}(y, \mathbf{x})-\hat{\eta}_{0, n}(y, \mathbf{x})
$$

where, for $t \in\{0,1\}$,

$$
\begin{aligned}
\hat{\eta}_{t, n}(y, \mathbf{x}) & =\hat{\xi}_{t, n}(Q, \mathbf{X}, T ; y, \mathbf{x}) \hat{\gamma}_{t, 0, n}(Q) \delta_{t}+\hat{\gamma}_{t, 1, n}(Q)(1-\delta)-\hat{\gamma}_{t, 2, n}(Q), \\
\hat{\gamma}_{t, 0, n}(\bar{y}) & =\exp \left\{\int_{0}^{\bar{y}-} \frac{\hat{H}_{t, 0, n}(d \bar{w})}{1-\hat{H}_{t, n}(\bar{w})}\right\} \\
\hat{\gamma}_{t, 1, n}(\bar{y}) & =\frac{1}{1-\hat{H}_{t, n}(\bar{y})} \int 1\{\bar{y}<\bar{w}\} \hat{\xi}_{t, n}(\bar{w}, \overline{\mathbf{x}}, t ; y, \mathbf{x}) \hat{\gamma}_{t, 0, n}(\bar{w}) \hat{H}_{t, 11, n}(d \bar{w}, d \overline{\mathbf{x}}), \\
\hat{\gamma}_{t, 2, n}(\bar{y}) & =\iint \frac{1\{\bar{v}<\bar{y}, \bar{v}<\bar{w}\} \hat{\xi}_{t, n}(\bar{w}, \overline{\mathbf{x}}, t ; y, \mathbf{x})}{\left[1-\hat{H}_{t, n}(\bar{v})\right]^{2}} \hat{\gamma}_{t, 0, n}(\bar{w}) \hat{H}_{t, 0, n}(d \bar{v}) \hat{H}_{t, 11, n}(d \bar{w}, d \overline{\mathbf{x}}),
\end{aligned}
$$

where $\hat{\xi}_{1, n}(\cdot, \cdot, \cdot ; y, \mathbf{x})$ and $\hat{\xi}_{0, n}(\cdot, \cdot, \cdot ; y, \mathbf{x})$ are defined as in (3.4) and (3.5), respectively, but with the true propensity score $p_{0}(\cdot)$ replaced by its SLE $\hat{p}_{n}(\cdot)$, and

$$
\begin{aligned}
\hat{H}_{t, n}(\bar{w}) & =\frac{1}{n} \sum_{i=1}^{n} 1\left\{Q_{i} \leq \bar{w}\right\} 1\left\{T_{i}=t\right\}, \\
\hat{H}_{t, 0, n}(\bar{w}) & =\frac{1}{n} \sum_{i=1}^{n}\left(1-\delta_{i}\right) 1\left\{Q_{i} \leq \bar{w}\right\} 1\left\{T_{i}=t\right\}, \\
\hat{H}_{t, 11, n}(\bar{w}, \overline{\mathbf{x}}) & =\frac{1}{n} \sum_{i=1}^{n} \delta_{i} 1\left\{Q_{i} \leq \bar{w}\right\} 1\left\{\mathbf{X}_{i} \leq \overline{\mathbf{x}}\right\} 1\left\{T_{i}=t\right\},
\end{aligned}
$$

are the sample counterparts of $H_{t}(\bar{w}), H_{t, 0}(\bar{w})$ and $H_{t, 11}(\bar{w}, \overline{\mathbf{x}})$, respectively.

Finally, we must consider nonparametric estimators for $\alpha(\cdot ; y, \mathbf{x})=\alpha_{1}(\cdot ; y, \mathbf{x})-\alpha_{0}(\cdot ; y, \mathbf{x})$, $\alpha_{1}(\cdot ; y, \mathbf{x})$ and $\alpha_{1}(\cdot ; y, \mathbf{x})$ being defined in (3.8). To this end, we must estimate $F_{Y(0) \mid \mathbf{x}}(y \mid \cdot)$ and $F_{Y(1) \mid \mathbf{X}}(y \mid \cdot)$. In the absence of censored data, Donald and Hsu (2014) propose estimating these functions using nonparametric series regression. Given that the outcome of interest $Y$ is subjected to censoring, such a procedure is not at our disposal. Notwithstanding, by using the Kaplan-Meier weights as discussed in Sections 2 and 3.1, we can overcome such a problem and 
estimate $F_{Y(0) \mid \mathbf{X}}(y \mid \mathbf{x})$ and $F_{Y(1) \mid \mathbf{X}}(y \mid \mathbf{x})$ by the Kaplan-Meier series estimators:

$$
\begin{aligned}
\hat{F}_{Y(0) \mid \mathbf{X}, n}^{k m}(y \mid \mathbf{x})=\left(\frac{n_{0}}{n} \sum_{i=1}^{n_{0}} W_{i n_{0}} \frac{1\left\{Q_{\left[i: n_{0}\right]} \leq y\right\}}{1-\hat{p}_{n}\left(\mathbf{X}_{\left[i: n_{0}\right]}\right)} \mathbf{R}^{L}\left(\mathbf{X}_{\left[i: n_{0}\right]}\right)\right)^{\prime} & \left(\frac{1}{n} \sum_{i=1}^{n} \mathbf{R}^{L}\left(\mathbf{X}_{i}\right) \mathbf{R}^{L}\left(\mathbf{X}_{i}\right)^{\prime}\right)^{-1} \mathbf{R}^{L}(\mathbf{x}),
\end{aligned}
$$

and

$$
\begin{aligned}
& \hat{F}_{Y(1) \mid \mathbf{X}, n}^{k m}(y \mid \mathbf{x})=\left(\frac{n_{1}}{n} \sum_{i=1}^{n_{1}} W_{i n_{1}} \frac{1\left\{Q_{\left[i: n_{1}\right]} \leq y\right\}}{\hat{p}_{n}\left(\mathbf{X}_{\left[i: n_{1}\right]}\right)} \mathbf{R}^{L}\left(\mathbf{X}_{\left[i: n_{1}\right]}\right)\right)^{\prime} \\
&\left(\frac{1}{n} \sum_{i=1}^{n} \mathbf{R}^{L}\left(\mathbf{X}_{i}\right) \mathbf{R}^{L}\left(\mathbf{X}_{i}\right)^{\prime}\right)^{-1} \mathbf{R}^{L}(\mathbf{x}),
\end{aligned}
$$

where $\mathbf{R}^{L}(\cdot)$ is the same power series used in the SLE estimator, with a potentially different number of series. Armed with (3.13) and (3.14), we can estimate $\alpha(\cdot ; y, \mathbf{x})$ by

$$
\hat{\alpha}_{n}^{k m}(\mathbf{X} ; y, \mathbf{x})=-\left(\frac{\hat{F}_{Y(1) \mid \mathbf{X}, n}^{k m}(y \mid \mathbf{X})}{\hat{p}_{n}(\mathbf{X})}+\frac{\hat{F}_{Y(0) \mid X, n}^{k m}(y \mid \mathbf{X})}{1-\hat{p}_{n}(\mathbf{X})}\right) 1\{\mathbf{X} \leq \mathbf{x}\} .
$$

Once we have nonparametric estimators for $p_{0}(\cdot), \eta(y, \mathbf{x})$, and $\alpha(\cdot ; y, \mathbf{x})$, the bootstrapped version of $\hat{I}_{1, n}(y, \mathbf{x})$ is given by

$$
\hat{I}_{1, n}^{*}(y, \mathbf{x})=\frac{1}{n} \sum_{i=1}^{n}\left[\hat{\eta}_{i, n}(y, \mathbf{x})+\hat{\alpha}_{n}^{K M}\left(\mathbf{X}_{i} ; y, \mathbf{x}\right)\left(T_{i}-\hat{p}_{n}\left(\mathbf{X}_{i}\right)\right)\right] V_{i}
$$

where $\hat{\eta}_{n}(y, \mathbf{x})=\hat{\eta}_{1, n}(y, \mathbf{x})-\hat{\eta}_{0, n}(y, \mathbf{x})$, and the random variables $\left\{V_{i}\right\}_{i=1}^{n}$ are iid with bounded support, zero mean and variance one, being independently generated from the sample $\left\{\left(Q_{i}, \delta_{i}, T_{i}, \mathbf{X}_{i}\right)\right\}_{i=1}^{n}$. A popular example involves iid Bernoulli variables $\left\{V_{i}\right\}$ with $\mathbb{P}(V=1-\kappa)=\kappa / \sqrt{5}$ and $\mathbb{P}(V=\kappa)=1-\kappa / \sqrt{5}$, where $\kappa=(\sqrt{5}+1) / 2$, as suggested by Mammen (1993).

Replacing $\hat{I}_{1, n}(y, \mathbf{x})$ with $\hat{I}_{1, n}^{*}(y, \mathbf{x})$, we get the bootstrap versions of $K S_{n}$ and $C v M_{n}, K S_{n}^{*}$ and $C v M_{n}^{*}$, respectively. The asymptotic critical values are estimated by

$$
\begin{gathered}
c_{n, \alpha}^{K S, *} \equiv \inf \left\{c_{\alpha} \in[0, \infty): \lim _{n \rightarrow \infty} \mathbb{P}_{n}^{*}\left\{K S_{n}^{*}>c_{\alpha}\right\}=\alpha\right\}, \\
c_{n, \alpha}^{C v M, *} \equiv \inf \left\{c_{\alpha} \in[0, \infty): \lim _{n \rightarrow \infty} \mathbb{P}_{n}^{*}\left\{C v M_{n}^{*}>c_{\alpha}\right\}=\alpha\right\}
\end{gathered}
$$

where $\mathbb{P}_{n}^{*}$ means bootstrap probability, i.e. conditional on the sample $\left\{\left(Q_{i}, \delta_{i}, T_{i}, \mathbf{X}_{i}\right)\right\}_{i=1}^{n}$. In practice, $c_{n, \alpha}^{K S, *}$ and $c_{n, \alpha}^{C v M, *}$ are approximated as accurately as desired by $\left(K S_{n}^{*}\right)_{B(1-\alpha)}$ and $\left(C v M_{n}^{*}\right)_{B(1-\alpha)}$, the $B(1-\alpha)-t h$ order statistic from $B$ replicates $\left\{K S_{n}^{b, *}\right\}_{l=1}^{B}$ of $K S_{n}^{*}$ or $\left\{C v M_{n}^{b, *}\right\}_{l=1}^{B}$ of $C v M_{n}^{*}$, respectively. 
Theorem 4 Let Assumptions 2.1, 2.2, 3.1 and Assumptions A.2-A.6 stated in Appendix A hold. Assume $\left\{V_{i}\right\}_{i=1}^{n}$ are iid, independent of the sample $\left\{\left(Q_{i}, \delta_{i}, T_{i}, \mathbf{X}_{i}\right)\right\}_{i=1}^{n}$, bounded random variables with zero mean and variance one. Then, under the null hypothesis (2.1), any fixed alternative hypothesis, or under the local alternatives (3.12)

$$
\sqrt{n} \hat{I}_{1, n}^{*}(y, \mathbf{x}) \Rightarrow C_{\infty}(y, \mathbf{x})
$$

where $C_{\infty}$ is the same Gaussian process of Theorem 1 and $\underset{*}{\Rightarrow}$ denoting weak convergence in probability under the the bootstrap law (see Giné and Zinn (1990)).

The following algorithm presents a complete procedure to implement our bootstrap-based tests for the null hypothesis of zero conditional distributional treatment effect as in (3.10). We focus on the Cramér-von Mises test but the Kolmogorov-Smirnov test is formed analogously.

Algorithm 1 1. Construct an estimate for the propensity score using $\hat{p}_{n}(\mathbf{x})=\mathcal{L}\left(\mathbf{R}^{L}(x)^{\prime} \hat{\boldsymbol{\pi}}_{L}\right)$, with $\hat{\boldsymbol{\pi}}_{L}$ as defined in (2.9).

2. For all data points $(y, \mathbf{x})=\left(Y_{i}, \mathbf{X}_{i}\right), i=1, \ldots, n$, compute $\hat{I}_{1, n}(y, \mathbf{x})$ using $(2.13)$, and construct the test statistic $C v M_{n}$ statistics as in (2.16).

3. Draw a realization of $\left\{V_{i}\right\}_{i=1}^{n}$.

4. Compute $\hat{I}_{1, n}^{*}(y, \mathbf{x})$ as in (3.15) and form $C v M_{n}^{b, *}$ statistic as (2.16), but with $\hat{I}_{1, n}^{*}(y, \mathbf{x})$ playing the role of $\hat{I}_{1, n}(y, \mathbf{x})$.

5. Repeat steps 3-4B times to obtain $\left\{C v M_{n}^{b, *}\right\}_{b=1}^{B}$.

6. Construct $c_{n, \alpha}^{C v M, *}$ as the empirical $(1-a)$-quantile of the $B$ bootstrap draws of $C v M_{n}^{b, *}$.

7. If $C v M_{n}>c_{n, \alpha}^{C v M, *}$, reject the null hypothesis (3.10). Otherwise, do not reject (3.10).

\section{Some extensions of the basic setup}

\subsection{Testing for the Zero Conditional Average Treatment Effect}

So far, we have only discussed tests for the existence of distribution treatment effects. Although the proposed tests for zero conditional distribution treatment effect are able to detect a very broad set of alternative hypotheses, they are still not able to pin down the direction of the departure from the null. For instance, if we reject the null (2.1), we unfortunately do not know if the policy affects the conditional mean or, instead, any other particular feature 
of the outcome distribution (e.g., its 5th moment). Being able to differentiate such cases is important: policy makers may be in favor of implementing a job training that reduces the average unemployment durations, but may be more reluctant to implement such policy if there is evidence that it affects only the other higher order moments. Given the major role played by the average treatment effect, in this section we show how to adapt our DTE tests to focus on this particular TE measure.

Let $\Upsilon^{\text {cate }}(\mathbf{X}) \equiv \mathbb{E}[Y(1) \mid \mathbf{X}]-\mathbb{E}[Y(0) \mid \mathbf{X}]$ be the conditional average treatment effect. From Lemma 1, we have that identification of $\Upsilon^{\text {cate }}(\cdot)$ is not guaranteed unless the support of the censoring variable is larger than or equal to the support the potential outcomes of interest. Given that in follow-up studies such condition is usually violated, it may be more appropriate to focus on the restricted conditional average treatment effect (CATE),

$$
\Upsilon_{\bar{\tau}}^{\text {cate }}(\mathbf{X}) \equiv \mathbb{E}[Y(1) 1\{Y(1) \leq \bar{\tau}\} \mid \mathbf{X}]-\mathbb{E}[Y(0) 1\{Y(0) \leq \bar{\tau}\} \mid \mathbf{X}]
$$

see e.g. Zucker (1998). From Lemma 1 we know that $\Upsilon_{\bar{\tau}}^{\text {cate }}(\cdot)$ is nonparametrically identified for all $\bar{\tau} \leq \tau$.

We are concerned with the following hypothesis:

$$
H_{0}^{\text {cate }}: \Upsilon_{\bar{\tau}}^{\text {cate }}(\mathbf{X})=0 \text { a.s. }
$$

Under $H_{0}^{\text {cate }}$, the restricted average treatment effect (ATE) is equal to zero for all subpopulations defined by covariates. The alternative hypothesis $H_{1}^{\text {cate }}$ is the negation of the null $H_{0}^{\text {cate }}$.

From the same reasoning of Lemma 2, we can rewrite (4.1) as

$$
H_{0}^{\text {cate }}: I_{\bar{\tau}}^{\text {cate }}(\mathbf{x})=0 \text { a.e. in } \mathcal{X}_{X}
$$

where $I_{\bar{\tau}}^{\text {cate }}(\mathbf{x})=I_{\bar{\tau}}^{1, \text { cate }}(\mathbf{x})-I_{\bar{\tau}}^{0}(\mathbf{x})$, with

$$
I_{\bar{\tau}}^{t, \text { cate }}(\mathbf{x}) \equiv \mathbb{E}^{k m}\left[\frac{1\{T=t\} Q 1\{Q \leq \bar{\tau}\}}{\mathbb{P}(T=t \mid \mathbf{X})} 1\{\mathbf{X} \leq \mathbf{x}\}\right], t \in\{0,1\} .
$$

Then, following the same steps as in Section 2.3, our $K S$ type test statistic for hypothesis (4.1) is

$$
K S_{\bar{\tau}, n}^{\text {cate }}=\sup _{\mathbf{x} \in \mathcal{X}_{X}}\left|\sqrt{n} \hat{I}_{\bar{\tau}, n}^{\text {cate }}(\mathbf{x})\right|
$$

where $\hat{I}_{\bar{\tau}, n}^{\text {cate }}(y, \mathbf{x})$ is defined in (B.1) at the Appendix B. The discussion for the $C v M$ test is the same and is, therefore, omitted. Notice that when $\bar{\tau}=\tau, 1\{Q \leq \tau\}=1$ a.s. and, therefore, no user-chosen trimming is necessary. This is of particular importance because, in this case, we 
are using all the information about the average treatment effect available in the data. In the simulation and application in Sections 5 and 6, we follow exactly this convention.

Under similar conditions to those in Section 3, we can derive the asymptotic linear representation of $\sqrt{n} \hat{I}_{\bar{\tau}, n}^{\text {cate }}(\mathbf{x})$. Using an analogous procedure to the one described in Section 3.4, let $c_{\bar{\tau}, \alpha, n}^{\text {cate }, *}$ denote the bootstrap critical value of the $K S_{\bar{\tau}, n}^{\text {cate }}$.

Theorem 5 Suppose Assumptions 2.1-2.2 and Assumptions A.2, A.4, A.5, A.7 and A.8 stated in Appendix A are satisfied. Then, for a fixed $\bar{\tau} \leq \tau$,

1. Under $H_{0}^{\text {cate }}, \lim _{n \rightarrow \infty} \mathbb{P}_{n}\left\{K S_{\bar{\tau}, n}^{\text {cate }}>c_{\bar{\tau}, \alpha, n}^{\text {cate }, *}\right\}=\alpha$.

2. Under $H_{1}^{\text {cate }}, \lim _{n \rightarrow \infty} \mathbb{P}_{n}\left\{K S_{\bar{\tau}, n}^{\text {cate }}>c_{\bar{\tau}, \alpha, n}^{\text {cate }, *}\right\}=1$.

3. Under $H_{1, n}^{\text {cate }}: \Upsilon_{\bar{\tau}}^{\text {cate }}(\mathbf{X})=n^{-1 / 2} h_{\bar{\tau}}^{\text {cate }}(\mathbf{X})$ a.s., if $h_{\bar{\tau}}^{\text {cate }}(\cdot)$ is an integrable function, and the set $h_{\bar{\tau}, n}^{\text {cate }} \equiv\left\{\mathbf{x} \in \mathcal{X}_{X}: n^{-1 / 2} h_{\bar{\tau}}^{\text {cate }}(\mathbf{x}) \neq 0\right\}$ has positive Lebesgue measure, then $\lim _{n \rightarrow \infty} \mathbb{P}_{n}\left\{K S_{\bar{\tau}, n}^{\text {cate }}>c_{\bar{\tau}, \alpha, n}^{\text {cate }, *}\right\}>\alpha$.

The results in Theorem 5 are related to Crump et al. (2008). In the absence of censoring, Crump et al. (2008) propose a test for $H_{0}^{\text {cate }}$ based on smooth estimates of the conditional average treatment effect. In particular, they use a series approach to estimate $\mathbb{E}[Y(1) \mid \mathbf{X}]$ and $\mathbb{E}[Y(0) \mid \mathbf{X}]$ and then compare how close the smooth estimate of $\Upsilon^{\text {cate }}(\cdot)$ is to zero. Given that Crump et al. (2008) test is based on the "local approach", their test for $H_{0}^{\text {cate }}$ is not able to detect local alternatives of the type of $H_{1, n}^{\text {cate }}$ and may suffer from the "curse of dimensionality." This is in contrast with the results in Theorem 5. Finally, note that as highlighted in Remark 1, our tests naturally cover the case where researchers are interested in testing for zero CATE only for a subset of covariates $\mathbf{X}_{1}$ of all available characteristics $\mathbf{X}$, whereas Crump et al. (2008) test does not. Thus, one can see that even when censoring is not an issue, our proposal is attractive when compared to Crump et al. (2008).

\subsection{Testing for Homogeneous Conditional Average Treatment Effect}

In this section, we show how one can adapt our baseline framework to test whether there is heterogeneity in the (restricted) ATE with respect to observed characteristics. In simple terms, we want to assess whether individuals with different background characteristics have different ATE. Such hypothesis is particularly relevant for policy makers interested in extending 
a pilot program to a larger population; if there is strong evidence against the hypothesis of homogeneous effect, one may be more concerned with targeting the appropriate population who should receive the treatment, see e.g. Manski (2004) and Crump et al. (2008).

As in Section 4.1, we focus on the restricted CATE. We seek to test

$$
H_{0}^{\text {hom }}: \exists \Upsilon_{\bar{\tau}} \in \mathbb{R}: \Upsilon_{\bar{\tau}}^{\text {cate }}(\mathbf{X})=\Upsilon_{\bar{\tau}} \text { a.s.. }
$$

The alternative hypothesis $H_{1}^{\text {hom }}$ is the negation of $H_{0}^{\text {hom }}$.

Note that we can rewrite (4.2) as

$$
H_{0}^{\text {hom }}: I_{\bar{\tau}}^{\text {hom }}(\mathbf{x})=0 \text { a.e. in } \mathcal{X}_{X}
$$

where $I_{\bar{\tau}}^{\text {hom }}(\mathbf{x})=I_{\bar{\tau}}^{1, \text { hom }}(\mathbf{x})-I_{\bar{\tau}}^{0, \text { hom }}(\mathbf{x})$,

$$
I_{\bar{\tau}}^{t, \text { hom }}(\mathbf{x}) \equiv \mathbb{E}^{k m}\left[1\{T=t\}\left(\frac{Q 1\{Q \leq \bar{\tau}\}}{\mathbb{P}(T=t \mid \mathbf{X})}-(2 T-1) I_{\bar{\tau}}^{a t e}\right) 1\{\mathbf{X} \leq \mathbf{x}\}\right],
$$

$t \in\{0,1\}$, and $I_{\bar{\tau}}^{\text {ate }}$ is the restricted average treatment effect,

$$
I_{\bar{\tau}}^{\text {ate }} \equiv \mathbb{E}^{k m}\left[\frac{T Q 1\{Q \leq \bar{\tau}\}}{\mathbb{P}(T=1 \mid \mathbf{X})}\right]-\mathbb{E}^{k m}\left[\frac{(1-T) Q 1\{Q \leq \bar{\tau}\}}{\mathbb{P}(T=0 \mid \mathbf{X})}\right] .
$$

Based on this characterization of $H_{0}^{\text {hom }}$, our proposed test statistic for (4.2) is

$$
K S_{\bar{\tau}, n}^{\text {hom }}=\sup _{\mathbf{x} \in \mathcal{X}_{X}}\left|\sqrt{n} \hat{I}_{\bar{\tau}, n}^{\text {hom }}(\mathbf{x})\right|
$$

where $\hat{I}_{\bar{\tau}, n}^{\text {hom }}(\mathbf{x})$ is defined in (B.2) in Appendix B. Let $c_{\bar{\tau}, \alpha, n}^{\text {hom, }}$ denote the bootstrap critical value of the $K S_{\bar{\tau}, n}^{\text {hom }}$.

Theorem 6 Suppose Assumptions 2.1-2.2 and Assumptions A.2, A.4, A.5, A.7 and A.8 stated in Appendix A are satisfied. Then, for a fixed $\bar{\tau} \leq \tau$,

1. Under $H_{0}^{\mathrm{hom}}, \lim _{n \rightarrow \infty} \mathbb{P}_{n}\left\{K S_{\bar{\tau}, n}^{\mathrm{hom}}>c_{\bar{\tau}, \alpha, n}^{\mathrm{hom}, *}\right\}=\alpha$.

2. Under $H_{1}^{\mathrm{hom}}, \lim _{n \rightarrow \infty} \mathbb{P}_{n}\left\{K S_{\bar{\tau}, n}^{\mathrm{hom}}>c_{\bar{\tau}, \alpha, n}^{\mathrm{hom}, *}\right\}=1$.

3. Under $H_{1, n}^{\text {hom }}: \Upsilon_{\bar{\tau}}(\mathbf{x})-\Upsilon_{\bar{\tau}}=n^{-1 / 2} h_{\bar{\tau}}^{\text {hom }}(\mathbf{X})$ a.s., if $h_{\bar{\tau}}^{\text {hom }}(\cdot)$ is an integrable function, and the set $h_{\bar{\tau}, n}^{\text {hom }} \equiv\left\{\mathbf{x} \in \mathcal{X}_{X}: n^{-1 / 2} h_{\bar{\tau}}^{\text {hom }}(\mathbf{x}) \neq 0\right\}$ has positive Lebesgue measure, then $\lim _{n \rightarrow \infty} \mathbb{P}_{n}\left\{K S_{\bar{\tau}, n}^{\text {hom }}>c_{\bar{\tau}, \alpha, n}^{\text {hom }, *}\right\}>\alpha$.

The results in Theorem 6 are related to Crump et al. (2008), who also proposed a test for $H_{0}^{\text {hom }}$ in a context in which censoring is not present. The test in Crump et al. (2008) is not able to detect local alternatives of the type of $H_{1, n}^{\text {hom }}$, and is not suitable to assess the presence of ATE 
heterogeneity when the conditioning vector in (4.2) is $\mathbf{X}_{1}$, a strict subset of $\mathbf{X}$. As discussed in Remark 1, our test easily accommodates this situation. Given these attractive features, we argue that, even when censoring is not an issue, the results in Theorem 6 are of interest to applied researchers and policy makers.

\subsection{Testing within the Local Treatment Effect setup}

In many important applications, the assumption that treatment allocation is exogenous may be too restrictive. For instance, when individuals do not comply with their treatment assignment, or more generally when they sort into treatment based on expected gains, Assumption 2.1 is likely to be violated. The goal of this section is to show that, if the unconfoundedness assumption does not hold, our tests are still applicable to the local treatment effect (LTE) setup introduced by Imbens and Angrist (1994) and Angrist et al. (1996).

The LTE setup presumes the availability of a binary instrumental variable $Z$ for the treatment assignment. Denote $T(0)$ and $T(1)$ the value that $T$ would have taken if $Z$ is equal to zero or one, respectively. The realized treatment is $T=Z T(1)+(1-Z) T(0)$. Thus, the observed sample consists of $i$ id copies $\left\{\left(Q_{i}, \delta_{i}, T_{i}, Z_{i}, \mathbf{X}_{i}\right)\right\}_{i=1}^{n}$. Denote $q_{0}(\mathbf{X}) \equiv \mathbb{P}(Z=1 \mid \mathbf{X})$.

To identify the LTE for the subpopulation of compliers, that is, individuals who comply with their actual assignment of treatment and would have complied with the alternative assignment, we need the following assumptions.

Assumption 4.1 (i) $(Y(0), Y(1), T(0), T(1), C(0), C(1)) \Perp Z \mid \mathbf{X}$; (ii) for some $\varepsilon>0, \varepsilon \leq$ $q_{0}(\mathbf{X}) \leq 1-\varepsilon$ a.s. and $\mathbb{P}(T(1)=1 \mid \mathbf{X})>\mathbb{P}(T(0)=1 \mid \mathbf{X})$ a.s.; and (iii) $\mathbb{P}(T(1) \geq T(0) \mid \mathbf{X})$ $=1$ a.s.

Assumption 4.2 Assume that $(i)(Y(0), Y(1)) \Perp(C(0), C(1)) \mid T, Z$; and $(i i)$ for $t \in\{0,1\}$, $\mathbb{P}(Y(t) \leq C(t) \mid \mathbf{X}, T, Z, Y(t))=\mathbb{P}(Y(t) \leq C(t) \mid T, Z, Y(t))$ a.s..

Assumption 4.1 is standard in the literature, see e.g. Abadie (2002), Abadie (2003), Frölich (2007). Assumption 4.2 is analogous to Assumption 2.2 and is necessary owing to the censoring. It is important to notice that Assumption 4.2 does not restrict how treatment status and instruments affect the censoring variable, which is weaker than typical assumptions used in the literature, see e.g. Frandsen (2015).

Because treatment effects are allowed to be arbitrarily heterogeneous, one is only able to identify effects for the complier subpopulation, see e.g. Abadie (2003), Frandsen (2015) and 
Sant'Anna (2016). Let $\Upsilon^{\text {ldte }}(y \mid \mathbf{X}) \equiv F_{Y(1) \mid \mathbf{X}}(y \mid \mathbf{X}$, pop $=$ comp $)-F_{Y(0) \mid \mathbf{X}}(y \mid \mathbf{X}$, pop $=$ comp $)$. Thus, our goal is to test the null hypothesis

$$
H_{0}^{\text {ldte }}: \Upsilon^{\text {ldte }}(y \mid \mathbf{X})=0 \text { a.s. } \forall y \in[-\infty, \tau]
$$

against $H_{1}^{\text {ldte }}$, which is simply the negation of (4.3). The null (4.3) is analogous to (2.1) within the LTE setup. For conciseness, we concentrate our attention on $H_{0}^{\text {ldte }}$, but of course, we can also adapt the hypotheses discussed in Sections 4.1 and 4.2 to the LTE setup in a routine fashion. Such extensions are presented in the Supplementary Appendix.

In order to proceed, we must show that $\Upsilon^{\text {ldte }}(y \mid \cdot)$ can be written in terms of observables $(Q, \delta, T, Z, \mathbf{X})$. In the Supplementary Appendix, we show that this is the case by extending Lemma 1 to the LTE setup. Then, using the integrated moment approach analogous to Lemma 2 , we can show that $H_{0}^{\text {ldte }}$ is true if and only if

$$
I^{\text {ldte }}(y, \mathbf{x})=0 \text { a.e. in }[-\infty, \tau] \times \mathcal{X}_{X}
$$

where $I^{\text {ldte }}(y, \mathbf{x})=I^{1, \text { ldte }}(y, \mathbf{x})-I^{0, \text { ldte }}(y, \mathbf{x})$, and for $t \in\{0,1\}$,

$$
\begin{aligned}
I^{t, l d t e}(y, \mathbf{x}) \equiv(2 t-1)\left\{\mathbb{E}^{k m}\right. & {\left[\frac{1\{Q \leq y\}}{q_{0}(\mathbf{X})} 1\{\mathbf{X} \leq \mathbf{x}\} \mid T=t, Z=1\right] \mathbb{P}(T=t, Z=1) } \\
& \left.-\mathbb{E}^{k m}\left[\frac{1\{Q \leq y\}}{1-q_{0}(\mathbf{X})} 1\{\mathbf{X} \leq \mathbf{x}\} \mid T=t, Z=0\right] \mathbb{P}(T=t, Z=0)\right\}
\end{aligned}
$$

Then, following the discussion in Section 2.3, our $K S$ type test statistic for hypothesis (4.3) is

$$
K S_{n}^{\text {ldte }}=\sqrt{n} \sup _{(y, \mathbf{x}) \in \mathcal{W}}\left|\hat{I}_{n}^{\text {ldte }}(y, \mathbf{x})\right|
$$

where $\hat{I}_{n}^{\text {ldte }}(y, \mathbf{x})$ is defined in (B.3) in Appendix B. Let $c_{\alpha, n}^{\text {ldte,* }}$ denote the bootstrap critical value of the $K S_{n}^{l d t e}$.

Theorem 7 Suppose Assumptions 4.1-4.2 are satisfied. Further, suppose that for the subpopulation of compliers, Assumption A.2, A.3 and A.6 stated in the Appendix are satisfied and that $q_{0}$ and its SLE $\hat{q}_{n}$ satisfy the analogous of Assumptions A.4 and A.5. Then,

1. Under $H_{0}^{\text {ldte }}, \lim _{n \rightarrow \infty} \mathbb{P}_{n}\left\{K S_{n}^{\text {ldte }}>c_{\alpha, n}^{\text {ldte,* }}\right\}=\alpha$.

2. Under $H_{1}^{\text {ldte }}, \lim _{n \rightarrow \infty} \mathbb{P}_{n}\left\{K S_{n}^{\text {ldte }}>c_{\alpha, n}^{\text {ldte }, *}\right\}=1$.

3. Under $H_{1, n}^{\text {ldte }}: \Upsilon^{\text {ldte }}(y \mid \mathbf{X})=\frac{1}{\sqrt{n}} h^{\text {ldte }}(y, \mathbf{X})$ a.s. $\forall y \in[-\infty, \tau]$, if $h^{\text {ldte }}(\cdot, \cdot)$ is an integrable function and the set $h_{n}^{\text {ldte }} \equiv\left\{(y, \mathbf{x}) \in \mathcal{W}: n^{-1 / 2} h^{\text {ldte }}(y, \mathbf{x}) \neq 0\right\}$ has positive Lebesgue measure, then $\lim _{n \rightarrow \infty} \mathbb{P}_{n}\left\{K S_{n}^{\text {ldte }}>c_{\alpha, n}^{\text {ldte,* }}\right\}>\alpha$. 
The results of Theorem 7 are related to Abadie (2002). In the absence of censoring, Abadie (2002) proposes a test for the unconditional analogue of $H_{0}^{\text {ldte }}$. Of course, by taking $w(\mathbf{X}, \mathbf{x})=$ 1 a.s., we are back to Abadie (2002) proposal. Thus, one may interpret Theorem 7 as extensions of Abadie (2002) in two different dimensions: it allows for covariates and for randomly censored outcomes. We are not aware of other proposals that can accommodate either these features.

\section{Monte Carlo simulations}

In this section, we conduct a small-scale Monte Carlo exercise in order to study the finite sample properties of our test statistics for the null hypotheses (2.1), (4.1) and (4.2). The $\left\{V_{i}\right\}_{i=}^{n}$ used in the bootstrap implementations are independently generated as $V$ with $\mathbb{P}(V=1-\kappa)=$ $\kappa / \sqrt{5}$ and $\mathbb{P}(V=\kappa)=1-\kappa / \sqrt{5}$, where $\kappa=(\sqrt{5}+1) / 2$, as proposed by Mammen (1993). The bootstrap critical values are approximated by Monte Carlo using 1,000 replications and the simulations are based on 10,000 Monte Carlo experiments. We report rejection probabilities at the $5 \%$ significance level. Results for $10 \%$ and $1 \%$ significance levels are similar and available upon request.

We consider the following three designs:

$$
\begin{aligned}
\text { (i). } Y(0)=1+X+\varepsilon(0), Y(1)=1+X+\varepsilon(1), \\
C(0)=C(1) \sim a_{1}+b_{1} \times \text { Exponential }(1) ; \\
(i i) . \quad Y(0)=1+X+e(0), Y(1)=2+X+e(1), \\
C(0)=C(1) \sim a_{2}+b_{2} \times \text { Exponential }(1) ; \\
(\text { iii }) . \quad Y(0)=1+X+e(1), Y(1)=1+3 X+e(1), \\
C(0)=C(1) \sim a_{3}+b_{3} \times \text { Exponential }(1) ;
\end{aligned}
$$

where $X$ is distributed as $U[0,1]$, independently of $e(0), e(1), C(0)$ and $C(1), \varepsilon(0)$ and $\varepsilon(1)$ are independent standard normal random variables and the parameters $a$ and $b$ are chosen such that the percentage of censoring is equal to 0,10 or 30 percent in the whole sample. In all designs, $\mathbb{P}(T=1 \mid X)=\exp (-0.5 X) /(1+\exp (-0.5 X))$. When testing (2.1) and (4.1), Design (i) falls under the null, whereas Designs $($ ii $)$ - (iii) fall under the alternative. When testing (4.2), Designs $(i)$ - (ii) fall under the null and Design ( $i i i)$ falls under the alternative. We set $\bar{\tau}=\tau$ when testing (4.1) and (4.2), implying that we do not rely on any truncation.

We report the proportion of rejections for sample sizes $n=100,300$ and 500 . We estimate $p(\cdot)$ using the SLE: with $n=100$ we use $1, X$, with $n=300$ we use $1, X, X^{2}$ and with 
Table 1: Empirical rejection probabilities, in percentage points.

\begin{tabular}{|c|c|c|c|c|c|c|c|c|}
\hline$D G P$ & Censoring & $n$ & $K S_{n}$ & $C v M_{n}$ & $K S_{n}^{\text {cate }}$ & $C v M_{n}^{\text {cate }}$ & $K S_{n}^{h o m}$ & $C v M_{n}^{h o m}$ \\
\hline (i) & 0 & 100 & 5.38 & 5.27 & 5.33 & 4.97 & 5.42 & 4.91 \\
\hline (i) & 10 & 100 & 5.32 & 5.07 & 4.80 & 4.74 & 5.15 & 4.82 \\
\hline (i) & 30 & 100 & 3.79 & 5.46 & 4.07 & 4.35 & 3.72 & 3.92 \\
\hline$(i i)$ & 0 & 100 & 97.52 & 98.50 & 99.04 & 98.93 & 5.85 & 5.10 \\
\hline$(i i)$ & 10 & 100 & 97.27 & 98.43 & 95.24 & 94.40 & 4.70 & 4.54 \\
\hline$(i i)$ & 30 & 100 & 76.28 & 95.86 & 52.74 & 52.98 & 4.19 & 4.14 \\
\hline$(i i i)$ & 0 & 100 & 94.78 & 89.51 & 97.18 & 89.54 & 27.72 & 48.22 \\
\hline$(i i i)$ & 10 & 100 & 92.33 & 86.65 & 91.58 & 80.91 & 16.22 & 27.32 \\
\hline$(i i i)$ & 30 & 100 & 73.57 & 78.96 & 61.08 & 52.45 & 7.11 & 9.84 \\
\hline$(i)$ & 0 & 300 & 5.33 & 5.00 & 5.45 & 5.34 & 5.44 & 5.54 \\
\hline$(i)$ & 10 & 300 & 5.31 & 5.10 & 4.94 & 4.59 & 4.73 & 4.32 \\
\hline (i) & 30 & 300 & 4.34 & 5.48 & 3.99 & 4.44 & 3.79 & 4.28 \\
\hline$(i i)$ & 0 & 300 & 100 & 100 & 100 & 100 & 5.16 & 4.83 \\
\hline$(i i)$ & 10 & 300 & 100 & 100 & 100 & 100 & 4.74 & 4.68 \\
\hline$(i i)$ & 30 & 300 & 99.51 & 100 & 92.81 & 92.53 & 4.17 & 4.39 \\
\hline$(i i i)$ & 0 & 300 & 100 & 100 & 100 & 100 & 94.27 & 99.42 \\
\hline$(i i i)$ & 10 & 300 & 100 & 100 & 100 & 100 & 66.50 & 84.70 \\
\hline$(i i i)$ & 30 & 300 & 99.81 & 99.95 & 97.67 & 95.02 & 22.42 & 33.66 \\
\hline$(i)$ & 0 & 500 & 5.04 & 5.32 & 5.31 & 5.20 & 5.66 & 5.53 \\
\hline (i) & 10 & 500 & 5.21 & 4.93 & 5.17 & 4.95 & 5.02 & 4.61 \\
\hline (i) & 30 & 500 & 4.62 & 5.38 & 4.14 & 4.35 & 4.45 & 4.34 \\
\hline$(i i)$ & 0 & 500 & 100 & 100 & 100 & 100 & 5.61 & 5.13 \\
\hline$(i i)$ & 10 & 500 & 100 & 100 & 100 & 100 & 5.05 & 4.72 \\
\hline$(i i)$ & 30 & 500 & 99.96 & 100 & 98.77 & 98.53 & 4.42 & 4.79 \\
\hline$(i i i)$ & 0 & 500 & 100 & 100 & 100 & 100 & 100 & 100 \\
\hline$(i i i)$ & 10 & 500 & 100 & 100 & 100 & 99.99 & 91.41 & 97.76 \\
\hline$(i i i)$ & 30 & 500 & 99.99 & 100 & 99.78 & 99.22 & 39.03 & 53.51 \\
\hline
\end{tabular}

$n=500$ we use $1, X, X^{2}, X^{3}$ as power functions in the estimation procedure. The proportion of rejections for our tests are presented in Table 1. $K S_{n}$ and $C v M_{n}$ stand for the $K S$ and $C v M$ test statistics for the null of zero conditional distribution treatment effect. $K S_{n}^{\text {cate }}$ and $C v M_{n}^{\text {cate }}$ are the analogous test statistics for the null of zero conditional average treatment effect and $K S_{n}^{\text {hom }}$ and $C v M_{n}^{\text {hom }}$ for the null of homogeneous average treatment effect across covariate values.

We observe that our tests exhibit good size accuracy even when $n=100$. When the censoring level is $30 \%$, we have that the proposed tests have size below their nominal levels, but as we increase the sample size, such size distortions are minimized. With respect to power, our $K S$ and $C v M$ test statistics reach satisfactory levels for $n=100$, the only exception being when testing for homogeneous ATE with a censoring level of 30\%. Nonetheless, as the sample size increases, all tests present satisfactory power properties, regardless of the censoring level considered. As one should expect, the power of all tests increases with sample size, and 
decreases with the degree of censoring. Overall, these simulations show that the proposed bootstrap tests exhibit excellent finite sample properties.

\section{Illinois Reemployment Bonus Experiment}

In this section, we demonstrate that our proposed tests can be useful in practice. We analyze data from the Illinois Reemployment Bonus Experiments, which is freely available at the W.E. Upjohn Institute for Employment Research.

From mid-1984 to mid-1985, the Illinois Department of Employment Security conducted a social experiment to test the effectiveness of bonus offers in reducing the duration of insured unemployment. At the beginning of each claim, the experiment randomly divided newly unemployed people into three groups:

1. Job Search Incentive Group (JSI). The members of this group were told that they would qualify for a cash bonus of $\$ 500$, which was about four times the average weekly unemployment insurance benefits, if they found a full-time job within eleven weeks of benefits and if they held that job for at least four months. 4816 claimants were assigned to this group.

2. Hiring Incentive Group (HI). The members of this group were told that their employer would qualify for a cash bonus of $\$ 500$ if the claimant found a full-time job within eleven weeks of benefits and if they held that job for at least four months. 3963 claimants were assigned to this group.

3. Control Group. All claimants not assigned to the other groups. These members did not know that the experiment was taking place. 3952 individuals were assigned to this group.

An important aspect of the Illinois Reemployment Bonus Experiment is that participation was not mandatory. Once claimants were assigned to the treatment groups, they were asked if they would like to participate in the demonstration or not. For those selected to the Job Search Incentive group, $84 \%$ agreed to participate, whereas just $65 \%$ of the Hiring Incentive group agreed to participate.

Several studies including Woodbury and Spiegelman (1987), Meyer (1996), and Bijwaard and Ridder (2005) have analyzed the impact of the reemployment bonus on the unemployment duration measured by the number of weeks receiving unemployment insurance. Spells which reached the 
maximum amount of benefits or the state maximum number of weeks, 26, are censored, leading to censoring proportions of 38, 41 and 42 percent for the JSI, HI and the control group, respectively. Apart from the duration data, some information about claimants' background characteristics is also available: age, gender (Male $=1$ ), ethnicity (White $=1$ ), pre-unemployment earnings and the weekly unemployment insurance benefits amount. For a complete description of the experiment and the available dataset, see Woodbury and Spiegelman (1987).

Our goal in this application is to assess the effect of reemployment bonuses on unemployment duration. Given the differences between JSI and HI, we analyze these two treatments separately. That is, we consider two sub-samples: one with individuals who are in JSI or in the control group, and one with individuals who are in HI or in the control group. ${ }^{3}$ Furthermore, we consider two types of analysis. First, we consider an intention to treat (ITT) analysis, where $T=1$ if an individual is offered to participate in the demonstration, and $T=0$ if an individual was in the control group. In this case, we completely ignored the non-compliance with treatment allocations. Second, in an attempt to disentangle the effects of being offered and actually receiving treatment, we consider a local treatment effect analysis, using the random assignment as an instrumental variable.

Table 2 reports the results of all our proposed tests, based on 10,000 bootstrap replications. We consider the nulls of (a) zero conditional (local) DTE, (b) zero conditional (local) ATE, and $(c)$ homogeneous (local) ATE across covariate values. The conditioning vector considered consists of all available claimants characteristics described above.

To implement all tests, we estimate the propensity score $p_{0}(\cdot)$ and the instrument propensity score $q_{0}(\cdot)$ using the SLE where all covariates enter the model linearly. Given that the data comes from an experimental design, consistency of the propensity score models is guaranteed.

Let us start interpreting the results for the JSI sample. For both the ITT and LTE setup, we reject the null of zero conditional (local) DTE at the $5 \%$ level using either the KolmogorovSmirnov or the Cramér-von Mises test statistic. Such evidence suggests that offering a reemployment bonus to job-searchers has affected the distribution of unemployment duration.

3 Given that the allocation to each treatment arm is random, i.e., the data come from a randomized control trial, there is no loss of generality of restricting our attention to these two sub-samples. In cases where one has more than two treatment groups and selection into these groups is not completely random, we foresee that one would be able to combine the tools developed in this paper with those in Cattaneo (2010) and Ao et al. (2019), under the unconfounded setup. When treatment is endogenous, though, it is not yet clear how one can extend the analysis to accomodate multiple instruments; see Mogstad et al. (2019) for some recent results in this direction. We leave a detailed discussion of these extensions to future research. 
Table 2: Bootstrap p-values for different tests for treatment effect heterogeneity based on the Illinois bonus experiment.

\begin{tabular}{|c|c|c|c|c|}
\hline \multicolumn{5}{|c|}{ Intention to Treat } \\
\hline & \multicolumn{2}{|c|}{ Job Search Incentive Group } & \multicolumn{2}{|c|}{ Hiring Incentive Group } \\
\hline Null Hypothesis / Test type & $K S$ & $C v M$ & $K S$ & $C v M$ \\
\hline Zero Conditional DTE & 0.0001 & 0.0122 & 0.0618 & 0.1516 \\
\hline Zero Conditional ATE & 0.0335 & 0.0686 & 0.1533 & 0.2167 \\
\hline Homogeneous Conditional ATE & 0.0319 & 0.0992 & 0.5568 & 0.6505 \\
\hline \multicolumn{5}{|c|}{ Local Treatment Effects - Compliers } \\
\hline & \multicolumn{2}{|c|}{ Job Search Incentive Group } & \multicolumn{2}{|c|}{ Hiring Incentive Group } \\
\hline Null Hypothesis / Test type & $K S$ & $C v M$ & $K S$ & $C v M$ \\
\hline Zero Conditional Local DTE & 0.0001 & 0.0105 & 0.0598 & 0.1559 \\
\hline Zero Conditional Local ATE & 0.0386 & 0.0766 & 0.1589 & 0.2191 \\
\hline Homogeneous Conditional Local ATE & 0.5910 & 0.5851 & 0.9949 & 0.9725 \\
\hline
\end{tabular}

To shed some light on which part of the distribution is affected, we test the null of zero conditional (restricted) ATE as in (4.1). In the LTE setup, we consider the analogous null of zero conditional local (restricted) ATE

$$
H_{0}^{\text {clate }}: \Upsilon_{\bar{\tau}}^{\text {clate }}(\mathbf{X})=0 \text { a.s., }
$$

where

$$
\begin{aligned}
\Upsilon_{\bar{\tau}}^{\text {clate }}(\mathbf{X})= & \mathbb{E}[Y(1) 1\{Y(1) \leq \bar{\tau}\} \mid \mathbf{X}, \text { pop }=\text { comp }] \\
& -\mathbb{E}[Y(0) 1\{Y(0) \leq \bar{\tau}\} \mid \mathbf{X}, \text { pop }=\text { comp }]
\end{aligned}
$$

For details about how one can construct tests for $H_{0}^{\text {clate }}$, see Section S.1.1 of the Supplementary Appendix.

We set $\bar{\tau}=26$, so all the available data is used. From Table 2, we have that $H_{0}^{\text {cate }}$ and $H_{0}^{\text {clate }}$ are both rejected at the $5 \%$ level when using the $K S$ test and at the $10 \%$ level when using the $C v M$ test. Such evidence suggests that the reemployment bonus affected the average unemployment duration. However, we note that Sant'Anna (2016) unconditional (restricted) ATE and LATE estimators are -0.2221 and 1.9745, respectively, and both are not statistically significant at the $10 \%$ level. Thus, a researcher who relied only on "traditional" unconditional tests of a zero average effect would have missed the presence of treatment effects in this program.

When conditional (local) ATE is heterogeneous, it may be harder to identify the subpopulation of individuals that the treatment effect is non-zero. However, if the conditional ATE is homogeneous, the task is trivial. With this in mind, we test the null of homogenous conditional (restricted) ATE as in (4.2). In the LTE setup, we consider the analogous null of homogenous 
conditional local (restricted) ATE,

$$
H_{0}^{l \text { hom }}: \exists \Upsilon_{\bar{\tau}}^{l} \in \mathbb{R}: \Upsilon_{\bar{\tau}}^{\text {clate }}(\mathbf{X})=\Upsilon_{\bar{\tau}}^{l} \text { a.s.. }
$$

For details about how one can construct tests for $H_{0}^{l \text { hom }}$, see Section S.1.2 of the Supplementary Appendix.

As before, we set $\bar{\tau}=26$. For the ITT setup, the null of homogeneous conditional ATE is rejected at the $5 \%$ level when using the $K S$ test, and at the $10 \%$ level when using the $C v M$ test. When the endogeneity of the selection into treatment is taken into account, we fail to reject the null of homogenous conditional local (restricted) ATE at usual confidence levels. From these results, one concludes that the average treatment effect of being offered versus not being offered into the bonus experiment is heterogeneous. On the other hand, once we restrict our attention to the complier subpopulation, we fail to find enough evidence against the null of homogeneous ATE of actually participating in the JSI program versus not participating.

Next, we analyze the results for the HI sub-sample. Interesting enough, at the $5 \%$ level, we fail to reject each considered null hypothesis regardless of the test statistic used. This finding suggests that offering a reemployment bonus to the employer does not affect the time unemployed individuals take to find a job at all.

Overall, the results of our proposed tests suggest that offering an unemployment bonus to the job searcher was effective in changing the length of the unemployment spell. On the other hand, offering the bonus to the employer rather than to the job-searcher seems to be ineffective in changing the unemployment duration.

\section{Conclusion and Suggestions for further Work}

In this article, we proposed a variety of nonparametric tests for treatment effect heterogeneity that can accommodate randomly censored outcomes and endogenous treatment allocations. We derived the asymptotic properties of the proposed tests, and have proved that critical values can be easily computed via a relatively simple multiplier bootstrap procedure. Furthermore, in contrast to other proposals, we proved that our tests are able to detect local alternatives converging to the null at the parametric rate. Our Monte Carlo simulations show that our proposed tests have good finite sample properties. Finally, our empirical application concerning the effect of unemployment bonus on unemployment duration showed the feasibility and appeal of our tests in relevant scenarios. Given the desirable features of our tests and the importance 
of treatment effect heterogeneity in assessing external validity, we argue that the tests proposed in this article are important additions to the applied researcher's toolkit.

For concreteness and compatibility of the procedures for both duration and non-duration outcomes, we framed the article within the context of time-invariant treatment allocation, i.e. when treatment allocation happens at beginning of the duration spell. Nonetheless, when treatment allocation is dynamic, the results of this article still apply to testing for treatment effect heterogeneity between those individuals treated at time $t$ and those not yet treated at time $t$, as in Sianesi (2004). Once the treatment and control groups are defined, the implementation of our proposed tests are exactly the same as described in the text.

In the rest of this section, we discuss extensions of our proposed methodology to other situations of practical interest. For example, we note that researchers may be interested in tests for conditional stochastic dominance, or more generally, tests based on conditional moment inequalities, see e.g. Andrews and Shi (2013, 2017), Delgado and Escanciano (2013), Chang et al. (2015) and Hsu (2017). As discussed in Section 5.1 of Andrews and Shi (2013), pointwise asymptotics results do not provide good approximations to the finite-sample properties of test statistics in conditional moment inequality models. Thus, in order to consider tests based on conditional moment inequalities under random censoring, one should first establish a uniform in DGP Bahadur expansion of a suitable two-step Kaplan-Meier integrals; for setting without censoring, see e.g. Lemma 3.1 and Lemma 3.2 in Hsu (2017). However, as noted in Remark 2, establishing such uniform Bahadur representation for Kaplan-Meier integrals is very technically challenging because traditional empirical process tools such as those in chapter 2.8 of van der Vaart and Wellner (1996) and in chapter 10 of Dudley (2014) are not directly applicable to Kaplan-Meier processes. Indeed, to the best of our knowledge, no such result is yet available in the literature, even for the classical univariate Kaplan-Meier estimator for the $\mathrm{CDF}$, let alone more general classes of functions. In light of this observation, we argue that a formal treatment of tests based on conditional moment inequalities under random censoring is beyond the scope of this article and is left for future research.

Another interesting extension one may whish to pursue is constructing tests for treatment effect heterogeneity with randomly right-censored outcomes that impose less stringent conditions on the censoring mechanism than those allowed under Assumption 2.2. For instance, one may wish to assume that, for $t \in\{0,1\},(Y(0), Y(1)) \Perp(C(0), C(1)) \mid T, \mathbf{X}$, and propose tests based on conditional Kaplan-Meier estimators using procedures similar to Akritas 
(1994), González-Manteiga and Cadarso-Suárez (1994) and Lopez (2011). Although such an assumption is less restrictive than Assumption 2.2, constructing tests for treatment effect heterogeneity based on conditional Kaplan-Meier estimators would rely on an additional set of assumptions. For instance, it would $(a)$ require additional stronger smoothness and differentiability assumptions; (b) rule out empirically relevant situations with discrete duration data as in our empirical application; $(c)$ involve choosing additional tuning parameters to estimate the conditional CDF; $(d)$ and perhaps even more importantly, the statistical analysis would rely on truncation arguments, which in turn would exclude from the analysis some important classes of functions such as (conditional) average treatment effects. At the cost of restricting how covariates may affect the probability of being censored, the tests proposed in this article bypass all these additional challenges. Given that these two procedures rely on different non-nested assumptions, and involves a very different type of statistical analysis, it is hard to generally rank them. We leave such task for future research. 


\section{Appendix A: Technical Assumptions}

We first present the technical Assumptions needed for our main results. Let $\mathcal{C}_{b}\left(\mathbb{R}^{k}\right)$ be the space of all bounded, continuous, complex-valued functions on $\mathbb{R}^{k}$.

Assumption A.1 The class of functions $\mathcal{F}=\left\{w(\mathbf{X}, \mathbf{x}): \mathbf{x} \in \Pi \subset[-\infty, \infty]^{k}\right\}$ satisfy one of the following conditions:

(i) $\mathcal{F} \subset \mathcal{C}_{b}\left(\mathbb{R}^{k}\right)$ is a vector lattice that contains the constant functions and separates points of $\mathbb{R}^{k}$.

(ii) $\mathcal{F} \subset \mathcal{C}_{b}\left(\mathbb{R}^{k}\right)$ is an algebra that contains the constant functions and separates points of $\mathbb{R}^{k}$.

(iii) $\mathcal{F}=\left\{w\left(\mathbf{x}^{\prime} \mathbf{X}\right): \mathbf{x} \in \Pi \subset[-\infty, \infty]^{k}\right\}$ and $w$ is an analytic function that is nonpolynomial, where $\Pi$ is a compact set of $\mathbb{R}^{k}$ containing the origin.

(iv) $\mathcal{F}=\left\{1\left(\mathbf{X} \in B_{x}\right): \mathbf{x} \in \Pi \subset[-\infty, \infty]^{k}\right\}$ and $\left\{B_{x}\right\}_{x \in \mathcal{X}_{X}}$ is a separating class of Borel sets of $\mathbb{R}^{k}$.

Assumption A.1 states the conditions on $w$ such that Lemma 2 holds. These conditions are exactly the same as those in Escanciano (2006) Lemma 1.

\section{Assumption A.2}

(i) The support $\mathcal{X}_{X}$ of the $k$-dimensional covariate $\mathbf{X}$ is a Cartesian product of compact intervals, $\mathcal{X}_{X}=\prod_{j=1}^{k}\left[x_{l j}, x_{u j}\right]$;

(ii) The density of $\mathbf{X}$ is bounded, and bounded away from 0 on $\mathcal{X}_{X}$

Assumption A.3 For $t \in\{0,1\}, F_{Y(t) \mid X}(y \mid \mathbf{X}=\mathbf{x})$ is m-times continuously differentiable in $x$, for $\operatorname{all}(y, \mathbf{x}) \in \mathcal{X}_{Y} \times \mathcal{X}_{X}, m \geq k$.

Assumption A.4 For all $\mathbf{x} \in \mathbb{X}_{X}$, the propensity score $p_{0}(\mathbf{x})$ is continuously differentiable of order $s \geq 13 k$, where $k$ is the dimension of $X$.

Assumption A.5 The series logit estimator of $p_{0}(\mathbf{x})$ uses a power series with $L=a \cdot N^{v}$ for some $a>0$ and $1 /(s / k-2)<v<1 / 11$.

Assumption A.6 For $t \in\{0,1\}$, assume that, for all $(y, \mathbf{x}) \in \mathcal{W}$,

$$
\begin{array}{r}
\mathbb{E}\left[\left(1\{Q(t) \leq y\} 1\{\mathbf{X} \leq \mathbf{x}\} \gamma_{t, 0}(Q) \delta_{t}\right)^{2}\right]<\infty \\
\mathbb{E}\left[1\{Q(t) \leq y\} 1\{\mathbf{X} \leq \mathbf{x}\} C_{t}^{1 / 2}(Y)\right]<\infty
\end{array}
$$


where $\gamma_{t, 0}$ is defined as in (3.1),

$$
C_{t}(w)=\int_{-\infty}^{w-} \frac{G_{t}(d y)}{\left[1-H_{t}(y)\right]\left[1-G_{t}(y)\right]},
$$

and $G_{t}(w)=\mathbb{P}(C \leq w, T=t)$.

Assumption A.7 For $t \in\{0,1\}, E(Y(t) \mid \mathbf{X}=\mathbf{x})$ is m-times continuously differentiable in $x$, for all $\mathbf{x} \in \mathcal{X}_{X}, m \geq k$.

Assumption A.8 For $t \in\{0,1\}$, assume that, for all $(y, \mathbf{x}) \in \mathcal{W}$,

$$
\begin{gathered}
\mathbb{E}\left[\left(Q(t) 1\{\mathbf{X} \leq \mathbf{x}\} \gamma_{t, 0}(Q) \delta_{t}\right)^{2}\right]<\infty \\
\mathbb{E}\left[Q(t) 1\{\mathbf{X} \leq \mathbf{x}\} C_{t}^{1 / 2}(Y)\right]<\infty
\end{gathered}
$$

Similar assumptions have adopted by Hirano et al. (2003), Crump et al. (2008), Donald and Hsu (2014), among others. Assumptions A.2, A.3 and A.7 restrict the distribution of $\mathbf{X}$ and $Y(t)$ and requires that all covariates are continuous. Nonetheless, at the expense of additional notation, we can deal with the case where $\mathbf{X}$ has both continuous and discrete components by means of sample splitting based on the discrete covariates. In order to avoid cumbersome notation, we abstract from this point in the rest of the paper. Assumption A.4 requires sufficient smoothness of the propensity score, whereas Assumption A.5 restrict the rate at which additional terms are added to the series approximation of $p(\cdot)$, depending on the dimension of $\mathbf{X}$ and the number of derivatives of $p(\cdot)$. The restriction on the derivatives in Assumption A.4 guarantees the existence of a $v$ that satisfy the conditions in Assumption A.5. Assumptions A.6 and A.8 are standard with censored data; they guarantee that the variance of the Kaplan-Meier integral related to the DTE and ATE is finite, and their bias are $o\left(n^{-1 / 2}\right)$. See Stute (1996) and Chen and Lo (1997) for a detailed discussion.

\section{Appendix B: Details About the Tests of Section 4}

In Section 4, we discuss extensions of our basic setup. More formally, we proposed tests for the null of zero conditional average treatment effect and for the null of constant average treatment effect across subpopulations. Furthermore, we showed how one can modify the aforementioned tests to accommodate endogenous treatment allocation. In this Appendix, we provide more details on how to construct the test statistics.

As discussed in Section 4.1, to test the null of zero conditional average treatment effect

$$
H_{0}^{\text {cate }}: \Upsilon_{\bar{\tau}}^{\text {cate }}(\mathbf{X})=0 \text { a.s. }
$$


one can use the $K S$-type test

$$
K S_{\bar{\tau}, n}^{\text {cate }}=\sup _{\mathbf{x} \in \mathcal{X}_{X}}\left|\sqrt{n} \hat{I}_{\bar{\tau}, n}^{\text {cate }}(\mathbf{x})\right|
$$

where

$$
\hat{I}_{\bar{\tau}, n}^{\text {cate }}(y, \mathbf{x})=\hat{I}_{\bar{\tau}, n}^{1, \text { cate }}(x)-\hat{I}_{\bar{\tau}, n}^{0}(\mathbf{x})
$$

with

$$
\hat{I}_{\bar{\tau}, n}^{t, \text { cate }}(\mathbf{x})=\sum_{i=1}^{n_{t}} W_{i n_{t}} \frac{Q_{i: n_{t}} 1\left\{Q_{i: n_{t}} \leq \bar{\tau}\right\} 1\left(\mathbf{X}_{\left[i: n_{t}\right]} \leq \mathbf{x}\right)}{\hat{\mathbb{P}}_{n}\left(T=t \mid \mathbf{X}_{\left[i: n_{t}\right]}\right)}, t \in\{0,1\},
$$

$\hat{\mathbb{P}}_{n}(T=t \mid \mathbf{X})$ the Series Logit Estimator for $\mathbb{P}(T=t \mid \mathbf{X}), t \in\{0,1\}$.

To test the null of homogeneous average treatment effect,

$$
H_{0}^{\text {hom }}: \exists \Upsilon_{\bar{\tau}} \in \mathbb{R}: \Upsilon_{\bar{\tau}}^{\text {cate }}(\mathbf{X})=\Upsilon_{\bar{\tau}} \text { a.s.. }
$$

we propose test statistic

$$
K S_{\bar{\tau}, n}^{\mathrm{hom}}=\sup _{\mathbf{x} \in \mathcal{X}_{X}}\left|\sqrt{n} \hat{I}_{\bar{\tau}, n}^{\mathrm{hom}}(\mathbf{x})\right|
$$

where

$$
\hat{I}_{\bar{\tau}, n}^{\mathrm{hom}}(\mathbf{x})=\hat{I}_{\bar{\tau}, n}^{1, \text { hom }}(\mathbf{x})-\hat{I}_{\bar{\tau}, n}^{0, \text { hom }}(\mathbf{x})
$$

with, for $t \in\{0,1\}$,

$$
\hat{I}_{\bar{\tau}, n}^{t, h o m}(\mathbf{x})=\frac{n_{t}}{n} \sum_{i=1}^{n_{t}} W_{i n_{t}}\left(\frac{Q_{i: n_{t}} 1\left\{Q_{i: n_{t}} \leq \bar{\tau}\right\}}{\hat{\mathbb{P}}_{n}\left(T=t \mid \mathbf{X}_{\left[i: n_{t}\right]}\right)}-(2 t-1) \hat{I}_{\bar{\tau}, n}^{a t e}\right) 1\left(\mathbf{X}_{\left[i: n_{t}\right]} \leq \mathbf{x}\right)
$$

$\hat{p}_{n}(\cdot)$ is the SLE for $p_{0}(\cdot)$, and

$$
\hat{I}_{\bar{\tau}, n}^{a t e}=\frac{n_{1}}{n} \sum_{i=1}^{n_{1}} W_{i n_{1}} \frac{Q_{i: n_{1}} 1\left\{Q_{i: n_{1}} \leq \bar{\tau}\right\}}{\hat{p}_{n}\left(\mathbf{X}_{\left[i: n_{1}\right]}\right)}-\frac{n_{0}}{n} \sum_{j=1}^{n_{0}} W_{j n_{0}} \frac{Q_{j: n_{0}} 1\left\{Q_{j: n_{0}} \leq \bar{\tau}\right\}}{1-\hat{p}_{n}\left(\mathbf{X}_{\left[j: n_{0}\right]}\right)} .
$$

Finally, to test the null of zero local conditional distribution treatment effect,

$$
H_{0}^{\text {ldte }}: \Upsilon^{\text {ldte }}(y \mid \mathbf{X})=0 \text { a.s. } \forall y \in[-\infty, \tau]
$$

we propose the $K S$ type test statistic

$$
K S_{n}^{l d t e}=\sqrt{n} \sup _{(y, \mathbf{x}) \in \mathcal{W}}\left|\hat{I}_{n}^{l d t e}(y, \mathbf{x})\right|
$$

where

$$
\hat{I}_{n}^{l d t e}(y, \mathbf{x})=\hat{I}_{n}^{1, l d t e}(y, \mathbf{x})-\hat{I}_{n}^{0, l d t e}(y, \mathbf{x})
$$

with

$$
\hat{I}_{n}^{1, \text { ldte }}(y, \mathbf{x})=\frac{n_{11}}{n} \sum_{i=1}^{n_{11}} W_{i n_{11}} \frac{1\left\{Q_{1: n_{11}} \leq y\right\} 1\left\{\mathbf{X}_{\left[i: n_{11}\right]} \leq \mathbf{x}\right\}}{\hat{q}_{n}\left(\mathbf{X}_{\left[i: n_{11}\right]}\right)}
$$




$$
\begin{aligned}
& -\frac{n_{10}}{n} \sum_{i=1}^{n_{10}} W_{i n_{10}} \frac{1\left\{Q_{1: n_{10}} \leq y\right\} 1\left\{\mathbf{X}_{\left[i: n_{10}\right]} \leq \mathbf{x}\right\}}{1-\hat{q}_{n}\left(\mathbf{X}_{\left[i: n_{10}\right]}\right)}, \\
\hat{I}_{n}^{0, l d t e}(y, \mathbf{x})= & \frac{n_{00}}{n} \sum_{j=1}^{n_{00}} W_{j n_{00}} \frac{1\left\{Q_{j: n_{00}} \leq y\right\} 1\left\{\mathbf{X}_{\left[j: n_{00}\right]} \leq \mathbf{x}\right\}}{1-\hat{q}_{n}\left(\mathbf{X}_{\left[j: n_{00}\right]}\right)} \\
& -\frac{n_{01}}{n} \sum_{j=1}^{n_{01}} W_{j n_{01}} \frac{1\left\{Q_{j: n_{01}} \leq y\right\} 1\left\{\mathbf{X}_{\left[j: n_{01}\right]} \leq \mathbf{x}\right\}}{\hat{q}_{n}\left(\mathbf{X}_{\left[j: n_{01}\right]}\right)}
\end{aligned}
$$

where $\hat{q}_{n}(\cdot)$ is the SLE for $q_{0}(\cdot), n_{t z}=\sum_{i=1}^{n} 1\{T=t\} 1\{Z=z\}, t, z \in\{0,1\}$, and for $1 \leq i \leq$ $n_{t z}, Q_{1: n_{t z}} \leq \cdots \leq Q_{n_{t z}: n_{t z}}$ are the ordered $Q$-values in the sub-sample with $\{T=t, Z=z\}$, $\mathbf{X}_{\left[i: n_{t z}\right]}$ and $\delta_{\left[i: n_{t z}\right]}$ are the $\mathbf{X}$ and $\delta$ paired with $Q_{i: n_{t z}}$, and

$$
W_{i n_{t z}}=\frac{\delta_{\left[i: n_{t z}\right]}}{n_{t z}-i+1} \prod_{j=1}^{i-1}\left[\frac{n_{t z}-j}{n_{t z}-j+1}\right]^{\delta_{\left[j: n_{t z}\right]}}
$$

is the Kaplan-Meier weights for the sub-sample with $\{T=t, Z=z\}$.

\section{References}

Abadie, A. (2002), "Bootstrap tests for distributional treatment effects in instrumental variable models," Journal of the American Statistical Association, 97(457), 284-292.

Abadie, A. (2003), "Semiparametric instrumental variable estimation of treatment response models," Journal of Econometrics, 113, 231-263.

Abrevaya, J., Hsu, Y.-C., and Lieli, R. P. (2015), "Estimating Conditional Average Treatment Effects," Journal of Business \&3 Economic Statistics, 33(4), 485-505.

Akritas, M. G. (1994), "Nearest neighbor estimation of a bivariate distribution under random censoring," The Annals of Statistics, 22(3), 1299-1327.

Andrews, D. W. K., and Shi, X. (2013), "Inference Based on Conditional Moment Inequalities," Econometrica, 81(2), 609-666.

Andrews, D. W., and Shi, X. (2017), "Inference based on many conditional moment inequalities," Journal of Econometrics, 196(2), 275-287.

Angrist, J. D., Imbens, G. W., and Rubin, D. B. (1996), "Identification of causal effects using instrumental variables," Journal of the American Statistical Association, 91(434), 444-455.

Anstrom, K. J., and Tsiatis, a. a. (2001), "Utilizing propensity scores to estimate causal treatment effects with censored time-lagged data.," Biometrics, 57(4), 1207-1218.

Ao, W., Calonico, S., and Lee, Y. Y. (2019), "Multivalued Treatments and Decomposition Analysis: An Application to the WIA Program," Journal of Business and Economic Statistics, 0(0), 1-31.

Bang, H., and Tsiatis, A. (2000), "Estimating medical costs with censored data," Biometrika, 87(2), 329-343.

Bierens, H. J. (1982), "Consistent model specification tests," Journal of Econometrics, 20(1982), 105-134.

Bierens, H. J., and Ploberger, W. (1997), "Asymptotic theory of integrated conditional moment tests," Econometrica, 65(5), 1129-1151.

Bijwaard, G. E., and Ridder, G. (2005), "Correcting for selective compliance in a re-employment bonus experiment," Journal of Econometrics, 125, 77-111.

Bitler, M. O., Gelbach, J. B., and Hoynes, H. H. (2006), "What mean impacts miss: Distributional effects of welfare reform experiments," The American Economic Review, 96(4), 988-1012. 
Bitler, M. O., Gelbach, J. B., and Hoynes, H. H. (2016), "Can Variation in Subgroups' Average Treatment Effects Explain Treatment Effect Heterogeneity? Evidence from a Social Experiment," Review of Economics and Statistics, .

Bitler, M. P., Gelbach, J. B., and Hoynes, H. W. (2008), "Distributional impacts of the Self-Sufficiency Project," Journal of Public Economics, 92(3-4), 748-765.

Blundell, R., and Powell, J. L. (2007), "Censored regression quantiles with endogenous regressors," Journal of Econometrics, 141(1), 65-83.

Cattaneo, M. D. (2010), "Efficient semiparametric estimation of multi-valued treatment effects under ignorability," Journal of Econometrics, 155(2), 138-154.

Chang, M., Lee, S., and Whang, Y.-J. (2015), "Nonparametric tests of conditional treatment effects with an application to single-sex schooling on academic achievements," The Econometrics Journal, 18(3), 307-346.

Chen, K., and Lo, S.-H. (1997), "On the rate of uniform convergence of the product-limit estimator: strong and weak laws," The Annals of Statistics, 25(3), 1050-1087.

Crump, R. K., Hotz, V. J., Imbens, G. W., and Mitnik, O. A. (2008), "Nonparametric tests for treatment effect heterogeneity," The Review of Economics and Statistics, 90(3), 389-405.

Delgado, M. a., and Escanciano, J. C. (2013), "Conditional stochastic dominance testing," Journal of Business 8 Economic Statistics, 31(1), 16-28.

Ding, P., Feller, A., and Miratrix, L. (2015), "Randomization inference for treatment effect variation," Journal of the Royal Statistical Society: Series B, .

Donald, S. G., and Hsu, Y.-C. (2014), "Estimation and inference for distribution functions and quantile functions in treatment effect models," Journal of Econometrics, 178(3), 383-397.

Dudley, R. M. (2014), Uniform Central Limit Theorems, second edn, Cambridge: Cambridge University Press.

Escanciano, J. C. (2006), "Goodness-of-Fit Tests for Linear and Nonlinear Time Series Models," Journal of the American Statistical Association, 101(474), 531-541.

Fan, Y., and Li, Q. (1996), "Consistent model specification tests: omitted variables and semiparametric functional forms," Econometrica, 64(4), 865-890.

Frandsen, B. R. (2015), "Treatment Effects With Censoring and Endogeneity," Journal of the American Statistical Association, 110(512), 1745-1752.

Frangakis, C. E., and Rubin, D. B. (1999), "Addressing complications of intention-to-treat analysis in the combined presence of all-or-none treatment-noncompliance and subsequent missing outcomes," Biometrika, $86(2), 365-379$.

Frölich, M. (2007), "Nonparametric IV estimation of local average treatment effects with covariates," Journal of Econometrics, 139(1), 35-75.

Giné, E., and Zinn, J. (1990), "Bootstrapping general empirical measures," The Annals of Probability, 18(2), 851-869.

González-Manteiga, W., and Cadarso-Suárez, C. (1994), "Asymptotic properties of a generalized kaplan-meier estimator with some applications," Journal of Nonparametric Statistics, 4(1), 65-78.

Hirano, K., Imbens, G. W., and Ridder, G. (2003), "Efficient estimation of average treatment effects using the estimated propensity score," Econometrica, 71(4), 1161-1189.

Honore, B., Khan, S., and Powell, J. (2002), "Quantile regression under random censoring," Journal of Econometrics, 109, 67-105.

Hotz, V. J., Imbens, G. W., and Mortimer, J. H. (2005), "Predicting the efficacy of future training programs using past experiences at other locations," Journal of Econometrics, 125, 241-270.

Hsu, Y.-C. (2017), "Consistent tests for conditional treatment effects," The Econometrics Journal, 20(1), 1-22. Imbens, G. W., and Angrist, J. D. (1994), "Identification and estimation of local average treatment effects," Econometrica, 62(2), 467-475. 
Imbens, G. W., and Wooldridge, J. M. (2009), "Recent developments in the econometrics of program evaluation," Journal of Economic Literature, 47(1), 5-86.

Kaplan, E. L., and Meier, P. (1958), "Nonparametric estimation from incomplete observations," Journal of the American Statistical Association, 53(282), 457-481.

Lee, M.-J. (2009), "Non-parametric tests for distributional treatment effect for randomly censored responses," Journal of the Royal Statistical Society: Series B (Statistical Methodology), 71(1), 243-264.

Lee, M.-J., and Lee, S.-J. (2005), "Analysis of job-training effects on Korean women," Journal of Applied Econometrics, 20(4), 549-562.

Lee, S., Okui, R., and Whang, Y.-J. J. (2017), "Doubly robust uniform confidence band for the conditional average treatment effect function," Journal of Applied Econometrics, 32(7), 1207-1225.

Lopez, O. (2011), "Nonparametric Estimation of the Multivariate Distribution Function in a Censored Regression Model with Applications," Communications in Statistics - Theory and Methods, 40(15), 2639-2660.

Mammen, E. (1993), "Bootstrap and wild bootstrap for high dimensional linear models," The Annals of Statistics, 21(1), 255-285.

Manski, C. F. (2004), "Statistical treatment rules for heterogeneous populations," Econometrica, 72(4), 12211246.

Meyer, B. D. (1996), "What have we learned from the Illinois reemployment bonus experiment?," Journal of Labor Economics, 14(1), 26-51.

Mogstad, M., Torgovitsky, A., and Walters, C. (2019), "Identification of Causal Effects with Multiple Instruments: Problems and Some Solutions," Working Paper, p. Working paper.

Rosenbaum, P. R., and Rubin, D. B. (1983), "The central role of the propensity score in observational studies for causal effects," Biometrika, 70(1), 41-55.

Sant'Anna, P. H. C. (2016), "Program Evaluation with Right-Censored Data," ArXiv preprint arXiv:1604.02642,

Sellero, C. S., González-Manteiga, W., and van Keilegom, I. (2005), "Uniform representation of product-limit integrals with applications," Scandinavian Journal of Statistics, 32, 563-581.

Shorack, G. R., and Wellner, J. A. (1986), Empirical processes with applications to statistics, New York: Wiley.

Sianesi, B. (2004), "An evaluation of the Swedish system of active labor market programs in the 1990s," The Review of Economics and Statistics, 86(1), 133-155.

Stinchcombe, M. B., and White, H. (1998), "Consistent specification testing with nuisance parameters present only under the alternative," Econometric theory, 14, 295-325.

Stute, W. (1993), "Consistent estimation under random censorship when covariables are present," Journal of Multivariate Analysis, 45, 89 - 103.

Stute, W. (1995), "The central limit theorem under random censorship," The Annals of Statistics, 23(2), 422439.

Stute, W. (1996), "Distributional convergence under random censorship when covariables are present," Scandinavian Journal of Statistics, 23(4), 461-471.

Stute, W. (1997), "Nonparametric model checks for regression," The Annals of Statistics, 25(2), 613-641.

Stute, W., and Wang, J.-L. (1993), "The strong law under random censorship," The Annals of Statistics, 21(3), 1591-1607.

van der Vaart, A. W., and Wellner, J. A. (1996), Weak Convergence and Empirical Processes, New York: Springer.

Woodbury, S. A., and Spiegelman, R. G. (1987), "Bonuses to workers and employers to reduce unemployment: Randomized trials in Illinois," The American Economic Review, 77(4), 513-530.

Zucker, D. M. (1998), "Restricted Mean Life With Covariates : Modification and Extension of a Useful Survival Analysis Method," Journal of the American Statistical Association, 93(442), 702-709. 